\title{
Study of Rayleigh-Bénard Convection of a Newtonian Nanoliquid in a High Porosity Medium Using Local Thermal Non-equilibrium Model
}

\section{P. G. Siddheshwar ${ }^{1}$. T. N. Sakshath ${ }^{1,2}$}

Published online: 7 November 2019

(c) Springer Nature India Private Limited 2019

\begin{abstract}
In the paper we make linear and non-linear stability analyses of Rayleigh-Bénard convection in a Newtonian, nanoliquid-saturated porous medium using local thermal non-equilibrium model (LTNE). The LTNE assumption results in advanced onset of convection and increase in heat transport when compared to that of local thermal equilibrium assumption. Freefree and rigid-rigid, isothermal boundaries are considered for investigation. The Galerkin method is used to obtain the critical eigen value. The influence of inter-phase heat transfer coefficient, ratio of thermal conductivities, Brinkman number, porous parameter on the onset of convection as well as on heat transport has been presented graphically and discussed in detail. The effect of increasing the value of porosity modified thermal conductivities advances the onset of convection and enhances the amount of heat transport whereas the remaining parameters have an opposing influence on both onset of convection as well as heat transport.
\end{abstract}

Keywords Rayleigh-Bénard convection · Nanoliquid · Porous medium · LTNE . Free-free $\cdot$ Rigid-rigid $\cdot$ Linear $\cdot$ Non-linear

\section{List of Symbols}

$\begin{array}{ll}A, B, C, D & \text { Amplitudes of convection } \\ C_{p} & \text { Specific heat at constant pressure } \\ d & \text { Channel depth } \\ \mathbf{g} & \text { Acceleration due to gravity } \\ h & \text { Inter-phase heat transfer coefficient } \\ H & \text { Dimensionless inter-phase heat transfer coefficient } \\ k & \text { Wavenumber in the } x \text { direction }\end{array}$

P. G. Siddheshwar

mathdrpgs@gmail.com

T. N. Sakshath

sakshath.tn@gmail.com

1 Department of Mathematics, Bangalore University, Jnana Bharathi Campus, Bangalore 560056, India

2 Department of Mathematics, Faculty of Mathematical and Physical Sciences, M S Ramaiah University of Applied Sciences, Peenya, Bangalore 560058, India 


$\begin{array}{ll}K & \text { Permeability } \\ p & \text { Pressure } \\ P r & \text { Prandtl number } \\ \vec{q} & \text { Filtration velocity or Darcy velocity } \\ t & \text { Time } \\ T & \text { Temperature } \\ V & \text { Dimensional velocity in the } y \text { direction } \\ x, z & \text { Cartesian coordinate } \\ X, Z & \text { Dimensionless coordinates }\end{array}$

\section{Greek Symbols}

$\begin{array}{ll}\alpha_{n l} & \text { Thermal diffusivity of the nanoliquid } \\ \alpha_{b l} & \text { Thermal diffusivity of the base liquid } \\ \gamma & \text { Porosity-modified ratio of thermal conductivities } \\ \beta & \text { Thermal expansion coefficient } \\ \mathrm{k} & \text { Thermal conductivity } \\ \Lambda & \text { Ratio of viscosities or Brinkman number } \\ \mu & \text { Viscosity of the nanoliquid } \\ \mu^{\prime} & \text { Viscosity of the nanoliquid in saturated porous medium } \\ \phi & \text { Porosity }(0<\phi<1) \\ \Psi & \text { Dimensionless stream function } \\ \psi & \text { Dimensional stream function } \\ \rho & \text { Density } \\ \sigma^{2} & \text { Inverse Darcy number or porous parameter } \\ \tau & \text { Dimensionless time } \\ \Theta & \text { Dimensionless temperature }\end{array}$

\section{Subscripts or Superscripts}

0

b

$c$

Reference value

Basic state

c Critical

bl Base liquid

$n l \quad$ Nano liquid

$L T E \quad$ Local thermal equilibrium

LTNE Local thermal non-equilibrium

$s$

Solid

\section{Introduction}

Thermal convection in fluid-saturated porous media is of interest with respect to many engineering and environmental applications, such as ice production, food cooling, crystal growth and others. The studies are mainly based on the assumption that the fluid and porous medium phases are everywhere in local thermal equilibrium (LTE) where the temperature gradient at any location between the two phases is assumed to be negligible. However, in many practical applications involving large temperature differences between the fluid and solid phases the assumption of LTE is inadequate. In such circumstances, it is pertinent to take account of the local thermal non-equilibrium (LTNE) effects by considering a two-field model for the energy equation, one each for the fluid and solid phases. The studies on convective flows in 
a porous medium considering LTNE effects is well documented in the book by Nield and Bejan [11].

The problem in a non-rotating porous medium using LTNE has been been investigated by many authors including Banu and Rees [3], Postelnicu and Rees [15], Rees and Pop [16], Straughan [20], Postelnicu [14], Nield and Kuznetsov [12], Barletta et al. [4], Nield et al. [13], Celli et al. [5], Siddheshwar and Siddabasappa [19] and many more. Asymptotic analyses of critical wave number, Rayleigh number and Nusselt number in the case of freefree and rigid-rigid, isothermal boundaries using LTNE is carried out by Siddheshwar and Siddabasappa [19].

Convection in a nanoliquid-saturated porous medium is also important as combination of both nanoliquids and porous medium can be used to regulate the residence time of heat in the system. Few papers have reported on the above problem $[1,2,18,19]$. In the present problem, we concentrate on the unconsidered aspects of the Brinkman-Bénard convection. Most of the works in literature consider the unrealistic free-free boundary conditions. The current study makes use of more realistic rigid-rigid boundaries. The limiting behaviour of $\mathrm{H}, \gamma$ and $\gamma H$ to obtain the results of LTE in case of rigid-rigid boundaries is clearly showcased in the present study through asymptotic analysis which is not seen in earlier works. This makes the problem more interesting and challenging than when considered under LTE assumption. There seems to be no work on Rayleigh-Bénard convection in a nanoliquid-saturated porous layer using LTNE model for free-free and rigid-rigid, isothermal boundaries

The objective of the paper is to investigate the following aspects in the current problem:

1. Studying the onset of Rayleigh-Bénard convection and amount of heat transport in a nanoliquid-saturated porous medium using free-free and rigid-rigid, isothermal boundaries.

2. Obtaining the results on Rayleigh-Bénard convection of Newtonian base liquids as a limiting case of the current problem.

3. Obtaining the results on Rayleigh-Bénard convection of Newtonian base liquids and nanoliquids using LTE assumption as a limiting case.

4. Regulating the residence time of heat in the system using nanoparticles and porous matrix.

5. Making predictions on the onset of convection as well as heat transport by varying various non-dimensional parameters.

6. Performing the asymptotic analysis of critical wave number, Rayleigh number and Nusselt number for small and large $\mathrm{H}$, large $\gamma$ and large $\gamma H$.

\section{Mathematical Formulation}

The schematic of the flow configuration is as shown in Fig. 1.

The governing equations for the problem are:

$$
\begin{aligned}
\nabla \cdot \vec{q} & =0, \\
\frac{\rho_{n l}}{\phi}\left(\frac{\partial \vec{q}}{\partial t}+\frac{1}{\phi}(\vec{q} \cdot \nabla) \vec{q}\right) & =-\nabla p+\mu^{\prime} \nabla^{2} \vec{q}-\frac{\mu}{K} \vec{q}+\rho_{n l} \vec{g}, \\
\left(\rho C_{p}\right)_{n l}\left(\phi \frac{\partial T_{n l}}{\partial t}+(\vec{q} \cdot \nabla) T_{n l}\right) & =\phi k_{n l} \nabla^{2} T_{n l}+h\left(T_{s}-T_{n l}\right), \\
(1-\phi)\left(\rho C_{p}\right)_{s} \frac{\partial T_{s}}{\partial t} & =(1-\phi) k_{s} \nabla^{2} T_{s}-h\left(T_{s}-T_{n l}\right), \\
\rho(T) & =\rho\left(T_{0}\right)\left[1-\beta_{n l}\left(T_{n l}-T_{0}\right)\right] .
\end{aligned}
$$




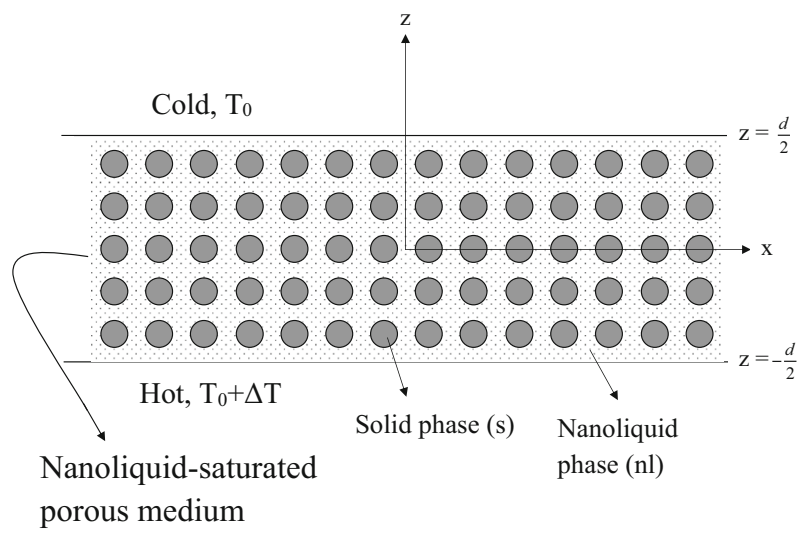

Fig. 1 Schematic of the physical configuration

Table 1 Thermophysical properties of 30\% glass fiber reinforced polycarbonate porous material at $300 \mathrm{~K}$ [8]

\begin{tabular}{lllll}
\hline $\begin{array}{l}\text { Density } \\
\left(\mathrm{kg} \mathrm{m}^{-3}\right)\end{array}$ & $\begin{array}{l}\text { Specific heat } \\
(\mathrm{J} / \mathrm{kg} \mathrm{K})\end{array}$ & $\begin{array}{l}\text { Thermal conduc- } \\
\text { tivity }(\mathrm{W} / \mathrm{m} \mathrm{K})\end{array}$ & $\begin{array}{l}\text { Thermal } \\
\text { diffusivity } \\
\left(\mathrm{m}^{2} \mathrm{~s}^{-1} \times 10^{7}\right)\end{array}$ & Porosity $(\phi)$ \\
\hline$(\rho)_{s}=1430$ & $\left(C_{p}\right)_{s}=1130$ & $k_{s}=0.24$ & $(\alpha)_{s}=1.4852$ & 0.88 \\
\hline
\end{tabular}

Table 2 Thermo-physical properties of ethylene glycol-copper saturated porous medium at $300 \mathrm{~K}$ for volume fraction, $\chi=0.06$ and $\phi=0.88$

\begin{tabular}{ll}
\hline Quantity & Ethylene glycol-copper saturated porous medium \\
\hline Density $(\rho)\left(\mathrm{kg} / \mathrm{m}^{3}\right)$ & 1565.09 \\
Specific heat $\left(C_{p}\right)(\mathrm{J} / \mathrm{kg} \mathrm{K})$ & 1662.34 \\
Thermal conductivity $(\mathrm{k})(\mathrm{W} / \mathrm{m} \mathrm{K})$ & 0.29294 \\
Thermal expansion coefficient $(\beta)\left(\mathrm{K}^{-1} \times 10^{5}\right)$ & 39.17174 \\
Dynamic coefficient of viscosity $\left(\mu^{\prime}\right)(\mathrm{kg} / \mathrm{m} \mathrm{s})$ & 0.02522673 \\
Thermal diffusivity $(\alpha)\left(\mathrm{m}^{2} \mathrm{~s}^{-1} \times 10^{7}\right)$ & 1.12545 \\
$\left(\rho C_{p}\right)\left(\mathrm{J} / \mathrm{m}^{3} \mathrm{~K} \times 10^{-6}\right)$ & 2.60172 \\
$(\rho \beta)\left(\mathrm{kg} / \mathrm{m}^{3} \mathrm{~K}\right)$ & 0.613073
\end{tabular}

We assume boundary conditions on $\vec{q}$ and $\mathrm{T}$ in the basic static state to be:

$$
\begin{gathered}
\vec{q}=0, T=T_{0}+\Delta T \text { at } \mathrm{z}=-\frac{d}{2}, \\
\vec{q}=0, T=T_{0} \text { at } \mathrm{z}=\frac{d}{2} .
\end{gathered}
$$

Further, the boundaries are assumed to be free-free or rigid-rigid. Taking the velocity, temperature, density and pressure in the quiescent basic state as follows:

$$
\left.\begin{array}{rl}
\vec{q} & =\vec{q}_{b}=(0,0), \\
T_{n l}(z) & =T_{n l_{b}}(z), \quad T_{s}(z)=T_{s_{b}}(z), \\
\rho(z) & =\rho_{b}(z), \quad p(z)=p_{b}(z),
\end{array}\right\},
$$



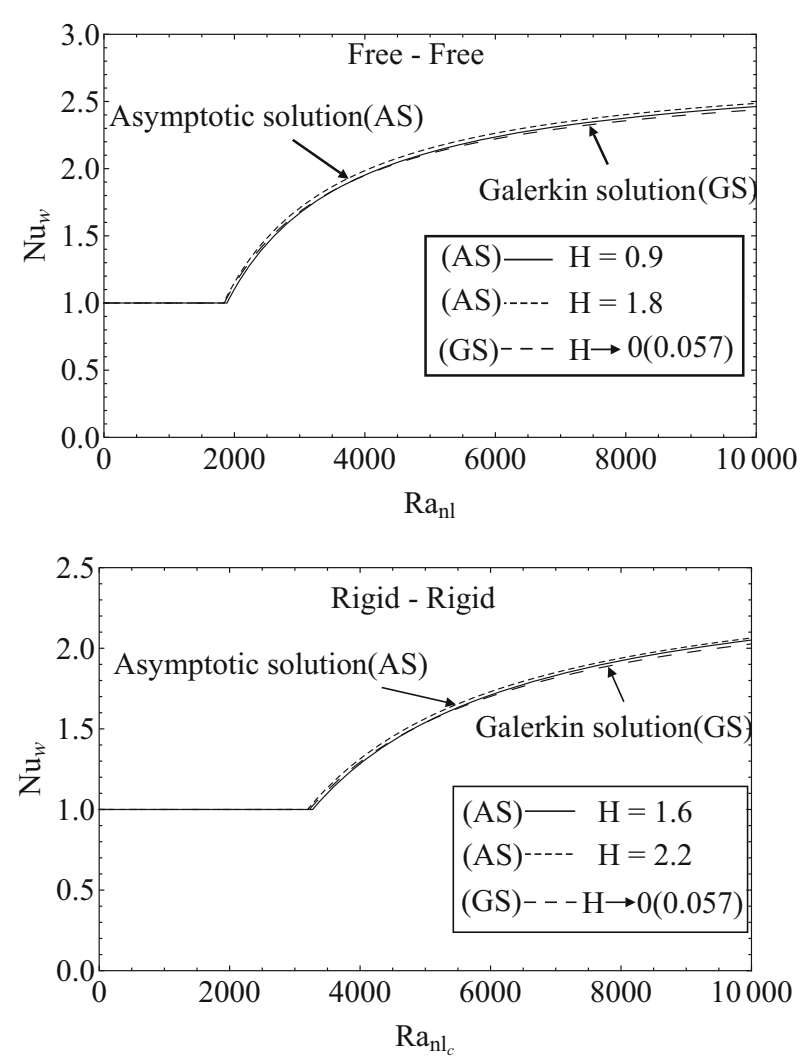

Fig. 2 Variation of $N u_{w}$ with $R a_{n l}$ of free-free and rigid-rigid boundaries, for $\Lambda=1.2, \sigma^{2}=5$ and $\gamma=10$, for small values of $\mathrm{H}$

we obtain the quiescent state solution for the temperature in the form:

$$
\begin{gathered}
T_{n l_{b}}(z)=T_{0}+\Delta T\left(\frac{1}{2}-\frac{z}{d}\right), \\
T_{s_{b}}(z)=T_{0}+\Delta T\left(\frac{1}{2}-\frac{z}{d}\right) .
\end{gathered}
$$

The other basic state quantities are not required explicitly in the analysis.

We now superimpose perturbation on the quiescent basic state and so we write:

$$
\left.\begin{array}{rl}
\vec{q} & =\vec{q}_{b}+\vec{q}^{\prime}(x, z, t), \\
T_{n l} & =T_{n l_{b}}+T^{\prime}(x, z, t), \\
T_{s} & =T_{s_{b}}+T^{\prime}(x, z, t), \\
\rho & =\rho_{b}+\rho^{\prime}(x, z, t), \\
p & =p_{b}+p^{\prime}(x, z, t),
\end{array}\right\},
$$

where the primes indicate a perturbed quantity. The pressure $p$ is eliminated between the $x$ and $y$-components of Eq. (2) and then the stream function $\psi(x, z, t)$ is introduced in the form 

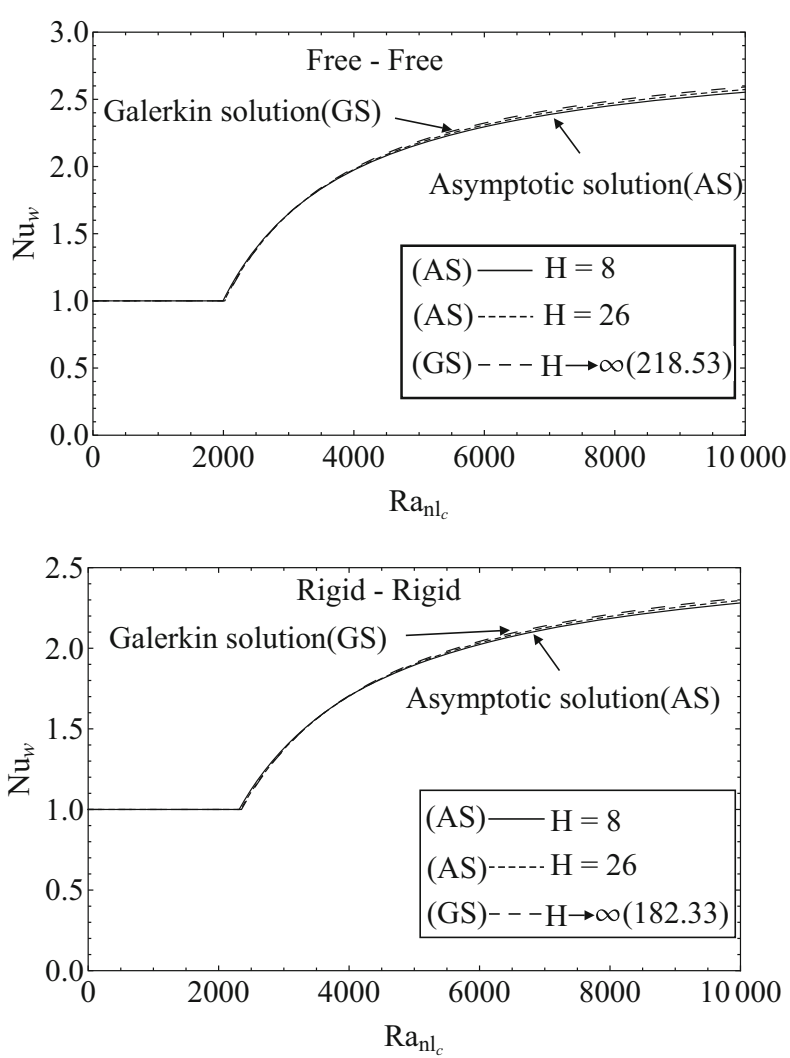

Fig. 3 Variation of $N u_{w}$ with $R a_{n l}$ of free-free and rigid-rigid boundaries, for $\Lambda=1.2, \sigma^{2}=5$ and $\gamma=10$, for large values of $\mathrm{H}$

$$
u=\frac{\partial \psi}{\partial z} \text { and } w=-\frac{\partial \psi}{\partial x} .
$$

The resulting equations are rendered dimensionless using the following scaling:

$$
\begin{aligned}
(X, Z) & =\left(\frac{x}{d}, \frac{z}{d}\right), V=\left(\frac{\left(\rho C_{p}\right)_{b l} d}{\phi k_{b l}}\right) v, \Psi=\left(\frac{\left(\rho C_{p}\right)_{b l}}{\phi k_{b l}}\right) \psi, \Theta_{n l}=\frac{T_{n l}}{\Delta T}, \\
\Theta_{s} & =\frac{T_{s}}{\Delta T}, \tau=\frac{k_{b l}}{\left(\rho C_{p}\right)_{b l} d^{2}} t .
\end{aligned}
$$

Using the scaling (10), the dimensionless form of the governing equations are:

$$
\begin{aligned}
& \frac{1}{P r_{n l}} \frac{\partial}{\partial \tau}\left(\nabla^{2} \Psi\right)=a_{1} \Lambda \nabla^{4} \Psi-a_{1} \sigma^{2} \nabla^{2} \Psi-a_{1}^{2} R a_{n l} \frac{\partial \Theta_{n l}}{\partial X}, \\
& \frac{\partial \Theta_{n l}}{\partial \tau}=-\frac{\partial \Psi}{\partial X}+a_{1} \nabla^{2} \Theta_{n l}+a_{1} H\left(\Theta_{s}-\Theta_{n l}\right)+\frac{\partial\left(\Psi, \Theta_{n l}\right)}{\partial(X, Z)}, \\
& \Gamma \frac{\partial \Theta_{s}}{\partial \tau}=a_{1} \nabla^{2} \Theta_{s}+a_{1} \gamma H\left(\Theta_{n l}-\Theta_{s}\right),
\end{aligned}
$$



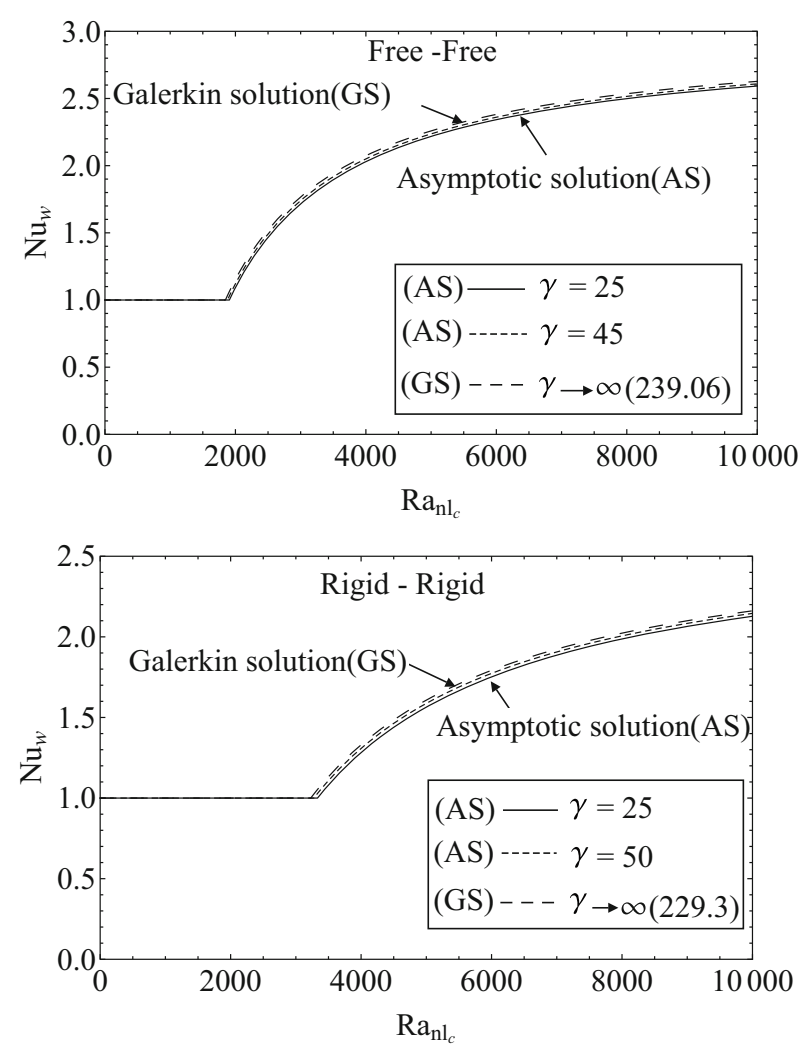

Fig. 4 Variation of $N u_{w}$ with $R a_{n l}$ of free-free and rigid-rigid boundaries, for $\Lambda=1.2, \sigma^{2}=5$ and $\mathrm{H}=$ 10 , for large values of $\gamma$

where

$$
\begin{aligned}
P r_{n l} & =\frac{\phi \mu_{n l}\left(\rho C_{p}\right)_{n l}}{\rho_{n l} k_{n l}}(\text { Prandtl number) } \\
a_{1} & =\frac{\alpha_{n l}}{\alpha_{b l}}(\text { ratio of thermal diffusivities) } \\
R a_{n l} & =\frac{(\rho \beta)_{n l}\left(\rho C_{p}\right)_{n l} g d^{3} \Delta T}{\phi k_{n l} \mu_{n l}} \text { (Rayleigh number), } \\
\Lambda & =\frac{\mu_{n l}^{\prime}}{\mu_{n l}}(\text { Brinkman number }), \sigma^{2}=\frac{d^{2}}{K} \text { (porous parameter), } \\
\Gamma & =\frac{k_{n l}\left(\rho C_{p}\right)_{s}}{k_{s}\left(\rho C_{p}\right)_{n l}}=F a_{1}, F=\frac{\alpha_{b l}}{\alpha_{s}} \\
H & =\frac{h d^{2}}{\phi k_{n l}}(\text { dimensionless inter-phase heat transfer coefficient), } \\
\gamma & =\frac{\phi k_{n l}}{(1-\phi) k_{s}} \text { (porosity modified ratio of thermal conductivities). }
\end{aligned}
$$



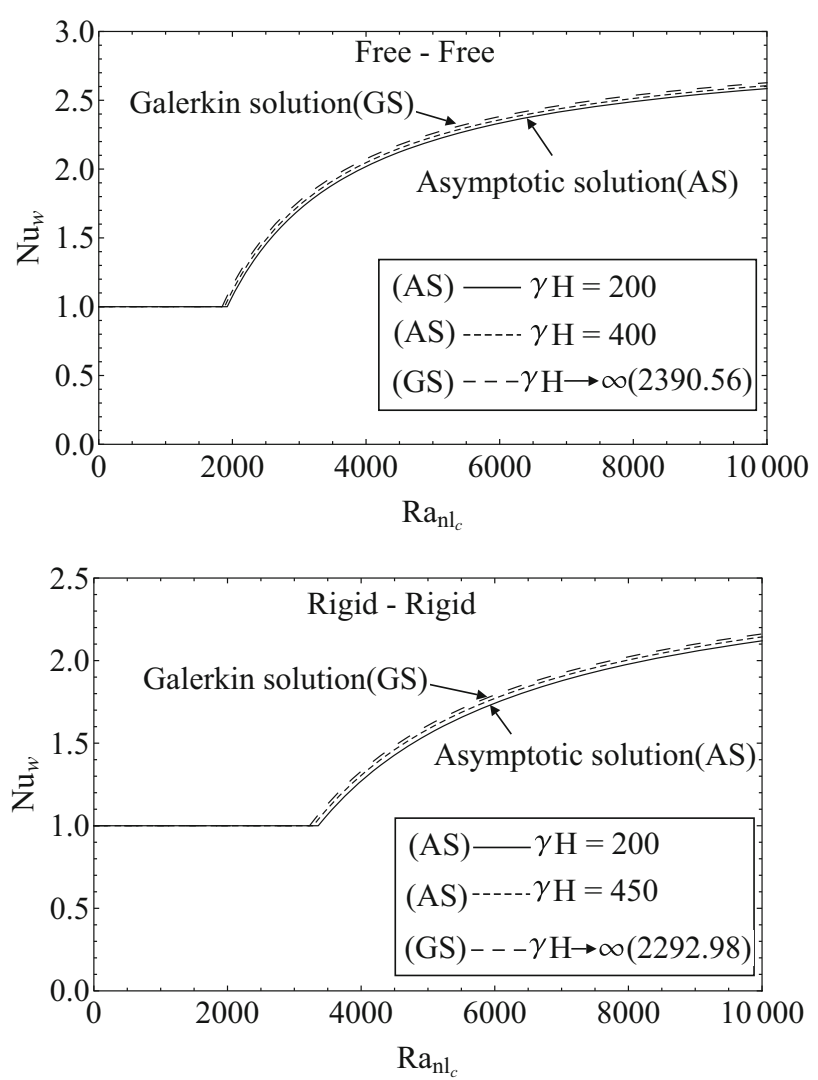

Fig. 5 Variation of $N u_{w}$ with $R a_{n l}$ of free-free and rigid-rigid boundaries, for $\Lambda=1.2, \sigma^{2}=5$ and $\mathrm{H}=$ 10 , for large values of $\gamma H$

We now carry out a linear stability analysis and study the onset of convection.

\section{Linear Stability Analysis for Marginal Stationary Convection for Free-Free/Rigid-Rigid, Isothermal Boundaries}

In order to make a linear stability analysis, we consider the linear and steady-state version of Eqs. (11)-(13).

\section{Free-Free, Isothermal Boundaries}

The boundary conditions suitable for free-free isothermal boundaries are:

$$
\Psi=\frac{\partial^{2} \Psi}{\partial Z^{2}}=\Theta_{n l}=\Theta_{s}=0 \text { at } Z= \pm \frac{1}{2} .
$$


Table 3 Comparison of exact and asymptotic solutions for small values of $\mathrm{H}$ and fixed values of $\gamma=0.01, \Lambda=1.2$ and $\sigma^{2}=5$ in case of free-free boundaries

Table 4 Comparison of exact and asymptotic solutions for large values of $\mathrm{H}$ and fixed values of $\gamma=10, \Lambda=1.2$ and $\sigma^{2}=5$ in case of free-free boundaries

\begin{tabular}{lllll}
\hline $\log _{10} H$ & $v_{c}(\mathrm{E})$ & $v_{c}(\mathrm{~A})$ & $R a_{n l c}(\mathrm{E})$ & $R a_{n l c}(\mathrm{~A})$ \\
\hline-3.5 & 2.61353 & 2.61353 & 1839.05 & 1839.05 \\
-3 & 2.61357 & 2.61357 & 1839.13 & 1839.13 \\
-2.5 & 2.61368 & 2.61368 & 1839.36 & 1839.36 \\
-2 & 2.61402 & 2.61402 & 1840.12 & 1840.12 \\
-1.5 & 2.61511 & 2.61511 & 1842.5 & 1842.5 \\
-1 & 2.61855 & 2.61855 & 1850.02 & 1850.02 \\
-0.5 & 2.62927 & 2.62927 & 1873.75 & 1873.75 \\
0 & 2.66175 & 2.6616 & 1948.25 & 1948.25 \\
0.5 & 2.75239 & 2.7483 & 2179.21 & 2179.22 \\
\hline
\end{tabular}

E-exact value, A-asymptotic value

\begin{tabular}{lllll}
\hline $\log _{10} H$ & $v_{c}(\mathrm{E})$ & $v_{c}(\mathrm{~A})$ & $R a_{n l c}(\mathrm{E})$ & $R a_{n l c}(\mathrm{~A})$ \\
\hline 1 & 2.62313 & 2.6224 & 1996.57 & 1997.31 \\
1.5 & 2.6172 & 2.61718 & 2013.69 & 2013.71 \\
2 & 2.61476 & 2.61476 & 2019.9 & 2019.9 \\
2.5 & 2.61392 & 2.61392 & 2021.95 & 2021.95 \\
3 & 2.61364 & 2.61364 & 2022.61 & 2022.61 \\
3.5 & 2.61356 & 2.61356 & 2022.82 & 2022.82 \\
4 & 2.61353 & 2.61353 & 2022.89 & 2022.89 \\
4.5 & 2.61352 & 2.61352 & 2022.91 & 2022.91 \\
5 & 2.61352 & 2.61352 & 2022.91 & 2022.91 \\
5.5 & 2.61352 & 2.61352 & 2022.92 & 2022.92 \\
6 & 2.61352 & 2.61352 & 2022.92 & 2022.92
\end{tabular}

E-exact value, A-asymptotic value

\begin{tabular}{lllll}
\hline $\log _{10} \gamma$ & $v_{c}(\mathrm{E})$ & $v_{c}(\mathrm{~A})$ & $R a_{n l c}(\mathrm{E})$ & $R a_{n l c}(\mathrm{~A})$ \\
\hline 1 & 2.62843 & 2.64175 & 1976.79 & 1961.24 \\
1.5 & 2.61577 & 2.61634 & 1891.61 & 1891.03 \\
2 & 2.61378 & 2.6138 & 1856.81 & 1856.79 \\
2.5 & 2.61354 & 2.61354 & 1844.77 & 1844.77 \\
3 & 2.61352 & 2.61352 & 1840.85 & 1840.85 \\
3.5 & 2.61352 & 2.61352 & 1839.6 & 1839.6 \\
4 & 2.61352 & 2.61352 & 1839.2 & 1839.2 \\
4.5 & 2.61352 & 2.61352 & 1839.07 & 1839.07 \\
5 & 2.61352 & 2.61352 & 1839.03 & 1839.03 \\
5.5 & 2.61352 & 2.61352 & 1839.02 & 1839.02 \\
6 & 2.61352 & 2.61352 & 1839.02 & 1839.02 \\
\hline
\end{tabular}

E-exact value, A-asymptotic value
Table 5 Comparison of exact and asymptotic solutions for large values of $\gamma$ and fixed values of $H=5, \Lambda=1.2$ and $\sigma^{2}=5$ in case of free-free boundaries 
Table 6 Comparison of exact and asymptotic solutions for large values of $\gamma H$ and fixed values of $H=10, \Lambda=1.2$ and $\sigma^{2}=5$ in case of free-free boundaries

\begin{tabular}{lllll}
\hline $\log _{10} \gamma H$ & $v_{c}(\mathrm{E})$ & $v_{c}(\mathrm{~A})$ & $R a_{n l c}(\mathrm{E})$ & $R a_{n l c}(\mathrm{~A})$ \\
\hline 2 & 2.62313 & 2.62763 & 1996.57 & 1992.14 \\
2.5 & 2.61475 & 2.61493 & 1894.25 & 1894.1 \\
3 & 2.61365 & 2.61366 & 1857.1 & 1857.1 \\
3.5 & 2.61353 & 2.61353 & 1844.8 & 1844.8 \\
4 & 2.61352 & 2.61352 & 1840.85 & 1840.85 \\
4.5 & 2.61352 & 2.61352 & 1839.6 & 1839.6 \\
5 & 2.61352 & 2.61352 & 1839.2 & 1839.2 \\
5.5 & 2.61352 & 2.61352 & 1839.07 & 1839.07 \\
6 & 2.61352 & 2.61352 & 1839.03 & 1839.03 \\
6.5 & 2.61352 & 2.61352 & 1839.02 & 1839.02 \\
7 & 2.61352 & 2.61352 & 1839.02 & 1839.02 \\
7.5 & 2.61352 & 2.61352 & 1839.02 & 1839.02 \\
8 & 2.61352 & 2.61352 & 1839.02 & 1839.02 \\
\hline
\end{tabular}

E-exact value, A-asymptotic value
Table 7 Comparison of exact and asymptotic solutions for small values of $\mathrm{H}$ and fixed values of $\gamma=0.01, \Lambda=1.2$ and $\sigma^{2}=5$ in case of rigid-rigid boundaries

\begin{tabular}{lllll}
\hline $\log _{10} H$ & $v_{c}(\mathrm{E})$ & $v_{c}(\mathrm{~A})$ & $R a_{n l c}(\mathrm{E})$ & $R a_{n l c}(\mathrm{~A})$ \\
\hline-3.5 & 3.15664 & 3.15664 & 3214.38 & 3214.38 \\
-3 & 3.15668 & 3.15668 & 3214.49 & 3214.49 \\
-2.5 & 3.15682 & 3.15682 & 3214.84 & 3214.84 \\
-2 & 3.15724 & 3.15724 & 3215.95 & 3215.95 \\
-1.5 & 3.15856 & 3.15856 & 3219.45 & 3219.45 \\
-1 & 3.16273 & 3.16273 & 3230.52 & 3230.52 \\
-0.5 & 3.17573 & 3.17572 & 3265.41 & 3265.41 \\
0 & 3.21515 & 3.21498 & 3374.81 & 3374.81 \\
0.5 & 3.32557 & 3.32073 & 3712.55 & 3712.56 \\
\hline
\end{tabular}

E-exact value, A-asymptotic value

The eigen solutions of the Eqs. (11)-(13) are:

$$
\left.\begin{array}{rl}
\Psi & =A \sin (v X) \sin \left[\pi\left(Z+\frac{1}{2}\right)\right], \\
\Theta_{n l} & =B \cos (v X) \sin \left[\pi\left(Z+\frac{1}{2}\right)\right], \\
\Theta_{s} & =C \cos (v X) \sin \left[\pi\left(Z+\frac{1}{2}\right)\right],
\end{array}\right\},
$$

where A, B and C are the amplitudes and $v$ is the wave number. Substituting Eq. (15) in the linearised version of Eqs. (11)-(13) and following the orthogonalisation procedure and rearrangement, we get

$$
R a_{n l_{c}}^{F F}=\frac{\delta_{c}^{4}\left(\delta_{c}^{2} \Lambda+\sigma^{2}\right)}{v_{c}^{2}}\left(1+\frac{H}{\delta_{c}^{2}+\gamma H}\right),
$$

where $\delta_{c}^{2}=v_{c}^{2}+\pi^{2}$. 
Table 8 Comparison of exact and asymptotic solutions for large values of $\mathrm{H}$ and fixed values of $\gamma=5, \Lambda=1.2$ and $\sigma^{2}=5$ in case of rigid-rigid boundaries

\begin{tabular}{lllll}
\hline $\log _{10} H$ & $v_{c}(E)$ & $v_{c}(A)$ & $R a_{n l c}(E)$ & $R a_{n l c}(A)$ \\
\hline 2 & 3.16415 & 3.16413 & 3832.64 & 3832.67 \\
2.5 & 3.15911 & 3.15911 & 3849.22 & 3849.23 \\
3 & 3.15742 & 3.15742 & 3854.65 & 3854.65 \\
3.5 & 3.15688 & 3.15688 & 3856.39 & 3856.39 \\
4 & 3.1567 & 3.1567 & 3856.94 & 3856.94 \\
4.5 & 3.15665 & 3.15665 & 3857.11 & 3857.11 \\
5 & 3.15663 & 3.15663 & 3857.17 & 3857.17 \\
5.5 & 3.15662 & 3.15662 & 3857.18 & 3857.18 \\
6 & 3.15662 & 3.15662 & 3857.19 & 3857.19 \\
6.5 & 3.15662 & 3.15662 & 3857.19 & 3857.19 \\
7 & 3.15662 & 3.15662 & 3857.19 & 3857.19 \\
7.5 & 3.15662 & 3.15662 & 3857.19 & 3857.19 \\
8 & 3.15662 & 3.15662 & 3857.19 & 3857.19 \\
\hline
\end{tabular}

E-exact value, A-asymptotic value
Table 9 Comparison of exact and asymptotic solutions for large values of $\gamma$ and fixed values of $H=5, \Lambda=1.2$ and $\sigma^{2}=5$ in case of rigid-rigid boundaries

\begin{tabular}{lllll}
\hline $\log _{10} \gamma$ & $v_{c}(E)$ & $v_{c}(A)$ & $R a_{n l c}(E)$ & $R a_{n l c}(A)$ \\
\hline 1 & 3.17998 & 3.20489 & 3482.47 & 3407.31 \\
1.5 & 3.16034 & 3.16145 & 3309.97 & 3303.21 \\
2 & 3.15706 & 3.1571 & 3245.84 & 3245.19 \\
2.5 & 3.15667 & 3.15667 & 3224.43 & 3224.36 \\
3 & 3.15663 & 3.15663 & 3217.53 & 3217.53 \\
3.5 & 3.15662 & 3.15662 & 3215.34 & 3215.34 \\
4 & 3.15662 & 3.15662 & 3214.65 & 3214.65 \\
4.5 & 3.15662 & 3.15662 & 3214.43 & 3214.43 \\
5 & 3.15662 & 3.15662 & 3214.36 & 3214.36 \\
5.5 & 3.15662 & 3.15662 & 3214.34 & 3214.34 \\
6 & 3.15662 & 3.15662 & 3214.33 & 3214.33 \\
\hline
\end{tabular}

E-exact value, A-asymptotic value

\section{Asymptotic Analysis}

Case 1: For small values of $\mathrm{H}$

We expand Ra given by Eq. (16) in a power series in $\mathrm{H}$ as

$$
R a_{n l}^{F F}=\frac{\delta^{4}\left(\delta^{2} \Lambda+\sigma^{2}\right)}{v^{2}}+\frac{\delta^{2}\left(\delta^{2} \Lambda+\sigma^{2}\right)}{v^{2}} H-\frac{\gamma\left(\delta^{2} \Lambda+\sigma^{2}\right)}{v^{2}} H^{2}+\cdots,
$$

where

$$
\delta^{2}=v^{2}+\pi^{2}
$$

Setting $\frac{\partial R a_{n l}^{F F}}{\partial v}=0$ for minimizing $R a_{n l}^{F F}$, we get:

$$
\begin{aligned}
& 4 \Lambda \nu^{6}+2 \nu^{4}\left(3 \pi^{2} \Lambda+\sigma^{2}\right)-2 \pi^{4}\left(\pi^{2} \Lambda+\sigma^{2}\right) \\
& +\left(2 \Lambda v^{4}-2 \pi^{2}\left(\pi^{2} \Lambda+\sigma^{2}\right)\right) H+2 \gamma\left(\pi^{2} \Lambda+\sigma^{2}\right) H^{2}=0
\end{aligned}
$$


Table 10 Comparison of exact and asymptotic solutions for large values of $\gamma H$ and fixed values of $H=10, \Lambda=1.2$ and $\sigma^{2}=5$ in case of rigid-rigid boundaries

\begin{tabular}{lllll}
\hline $\log _{10} \gamma H$ & $v_{c}(E)$ & $v_{c}(A)$ & $R a_{n l c}(E)$ & $R a_{n l c}(A)$ \\
\hline 2 & 3.17227 & 3.18075 & 3482.45 & 3471.78 \\
2.5 & 3.1587 & 3.15904 & 3309.97 & 3309.59 \\
3 & 3.15685 & 3.15686 & 3245.84 & 3245.83 \\
3.5 & 3.15665 & 3.15665 & 3224.43 & 3224.43 \\
4 & 3.15662 & 3.15662 & 3217.53 & 3217.53 \\
4.5 & 3.15662 & 3.15662 & 3215.34 & 3215.34 \\
5 & 3.15662 & 3.15662 & 3214.65 & 3214.65 \\
5.5 & 3.15662 & 3.15662 & 3214.43 & 3214.43 \\
6 & 3.15662 & 3.15662 & 3214.36 & 3214.36 \\
6.5 & 3.15662 & 3.15662 & 3214.34 & 3214.34 \\
7 & 3.15662 & 3.15662 & 3214.33 & 3214.33 \\
7.5 & 3.15662 & 3.15662 & 3214.33 & 3214.33 \\
8 & 3.15662 & 3.15662 & 3214.33 & 3214.33 \\
\hline
\end{tabular}

E-exact value, A-asymptotic value

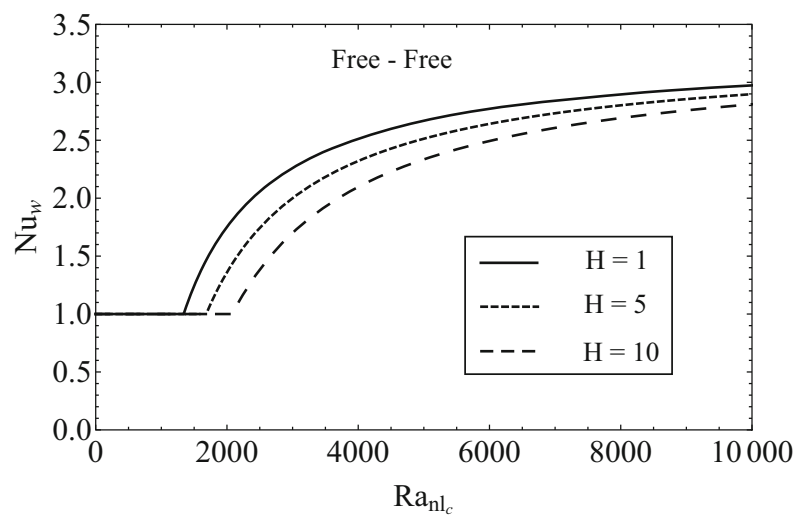

Fig. 6 Variation of weighted average Nusselt number, $N u_{w}$ with Rayleigh number, $R a_{n l}$, for different values of $\mathrm{H}$, for fixed values of $\Lambda=1.2, \sigma^{2}=5$ and $\gamma=0.1$

We also expand $v$ in terms of $\mathrm{H}$ as

$$
v=v_{0}+v_{1} H+v_{2} H^{2}+\cdots,
$$

where $v_{0}$ is the critical wave number for the LTE case which is given by

$$
v_{0}=\frac{1}{2} \sqrt{\frac{\sqrt{\left(\pi^{2} \Lambda+\sigma^{2}\right)\left(9 \pi^{2} \Lambda+\sigma^{2}\right)}-\pi^{2} \Lambda-\sigma^{2}}{\Lambda}} .
$$

Substituting Eq. (20) in Eq. (19) and equating the coefficients of like powers of H, we obtain $v_{1}$ and $v_{2}$ as:

$$
v_{1}=\frac{\Delta_{1}^{\prime}}{\Delta_{2}^{\prime}}
$$




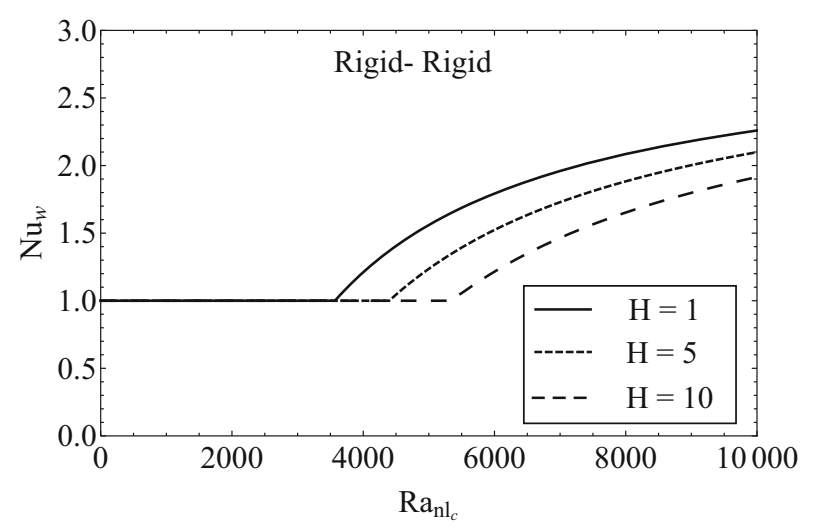

Fig. 7 Variation of $N u_{w}$ with Rayleigh number, $R a_{n l}$, for different values of $\mathrm{H}$, for fixed values of $\Lambda=1.2$, $\sigma^{2}=5$ and $\gamma=0.1$

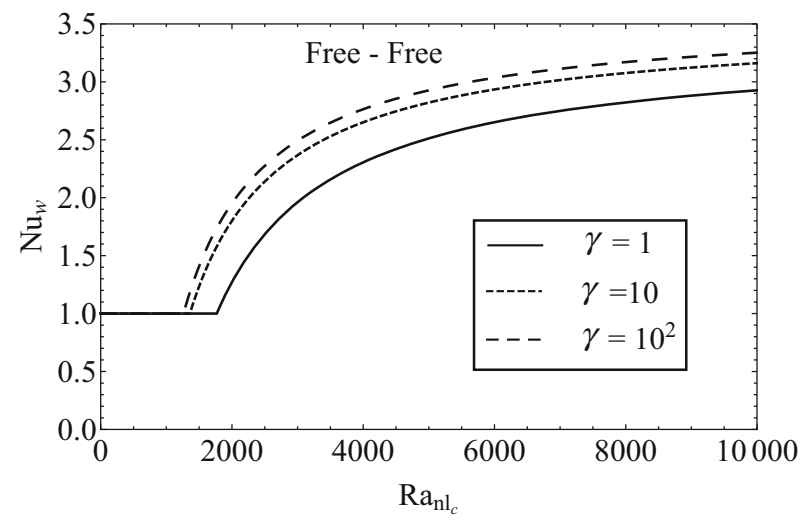

Fig. 8 Variation of $N u_{w}$ with $R a_{n l}$, for different values of $\gamma$, for fixed values of $\Lambda=1.2, \sigma^{2}=5$ and $\mathrm{H}=$ 10

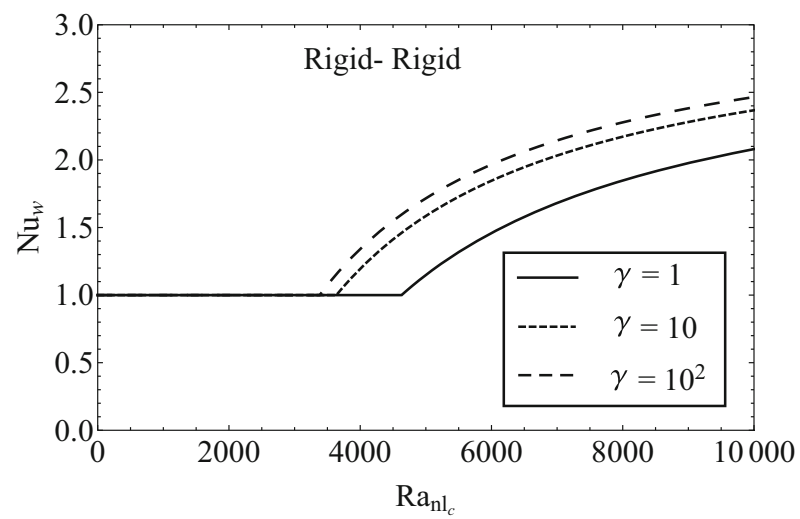

Fig. 9 Variation of $N u_{w}$ with $R a_{n l}$, for different values of $\gamma$, for fixed values of $\Lambda=1.2, \sigma^{2}=5$ and $\mathrm{H}=$ 10 


$$
v_{2}=\frac{\Delta_{3}^{\prime}}{\Delta_{2}^{\prime}}
$$

where

$$
\begin{aligned}
& \delta_{0}^{2}=v_{0}^{2}+\pi^{2} \\
& \Delta_{1}^{\prime}=\Lambda\left(\pi^{4}-v_{0}^{4}\right)+\pi^{2} \sigma^{2} \\
& \Delta_{2}^{\prime}=4 v_{0}^{3}\left(3 \delta_{0}^{2} \Lambda+\sigma^{2}\right) \\
& \Delta_{3}^{\prime}=\Lambda\left(-\pi^{2} \gamma-2 v_{1}\left(9 \pi^{2} \nu_{1}+v_{0}\left(15 v_{0} v_{1}+2\right)\right) v_{0}^{2}\right)+\sigma^{2}\left(\gamma+6 v_{0}^{2} v_{1}^{2}\right)
\end{aligned}
$$

Using the obtained values of $v_{0}, v_{1}, v_{2}$ in Eq. (20), we get the critical wave number and using this in the Eq. (17), we can obtain the critical Rayleigh number for small $\mathrm{H}$.

Case 2: For large values of $\mathrm{H}$

For large values of $\mathrm{H}, R a_{n l}^{F F}$ takes the form

$$
R a_{n l}^{F F}=\frac{(\gamma+1) \delta^{4}\left(\delta^{2} \Lambda+\sigma^{2}\right)}{\gamma v^{2}}+\frac{\delta^{8}\left(\delta^{2} \Lambda+\sigma^{2}\right)}{\gamma^{3} H^{2} v^{2}}-\frac{\delta^{6}\left(\delta^{2} \Lambda+\sigma^{2}\right)}{\gamma^{2} H v^{2}}+\cdots,
$$

where $\delta^{2}=v^{2}+\pi^{2}$.

Setting $\frac{\partial R a_{n l}^{F F}}{\partial v}=0$, we get:

$$
\begin{aligned}
& 2 \gamma^{2}(\gamma+1)\left(2 \Lambda v^{6}+v^{4}\left(3 \pi^{2} \Lambda+\sigma^{2}\right)-\pi^{4}\left(\pi^{2} \Lambda+\sigma^{2}\right)\right) \\
& \quad+2 \gamma \delta^{4}\left(\delta^{2} \Lambda\left(\pi^{2}-3 v^{2}\right)+\left(\pi^{2}-2 v^{2}\right) \sigma^{2}\right) \frac{1}{H} \\
& \quad+2 \delta^{6}\left(4 \Lambda v^{4}+3 v^{2}\left(\pi^{2} \Lambda+\sigma^{2}\right)-\pi^{2}\left(\pi^{2} \Lambda+\sigma^{2}\right)\right) \frac{1}{H^{2}}=0
\end{aligned}
$$

We expand $v$ in terms of $\frac{1}{H}<<1$ as

$$
v=v_{0}+\frac{v_{1}}{H}+\frac{v_{2}}{H^{2}}+\cdots,
$$

where $v_{0}$ is the critical wave number for the LTE case which is given by Eq. (21).

Substituting Eq. (30) in Eq. (29) and equating the coefficients of like powers of H, we obtain the values of $v_{1}$ and $v_{2}$ as:

$$
\begin{aligned}
& v_{1}=\frac{\delta_{0}^{4} \Delta_{4}^{\prime}}{\Delta_{5}^{\prime}}, \\
& v_{2}=\frac{\Delta_{6}^{\prime}}{\gamma \Delta_{5}^{\prime}},
\end{aligned}
$$

where

$$
\begin{aligned}
\Delta_{4}^{\prime}= & \left(2 v_{0}^{2}\left(\pi^{2} \Lambda+\sigma^{2}\right)+3 \Lambda v_{0}^{4}-\pi^{2}\left(\pi^{2} \Lambda+\sigma^{2}\right)\right) \\
\Delta_{5}^{\prime}= & 4 \gamma(\gamma+1) v_{0}^{3}\left(3 \delta_{0}^{2} \Lambda+\sigma^{2}\right) . \\
\Delta_{6}^{\prime}= & -6 \gamma^{2}(\gamma+1) v_{0}^{2} v_{1}^{2}\left(5 \Lambda v_{0}^{2}+3 \pi^{2} \Lambda+\sigma^{2}\right) \\
& +12 \gamma \delta_{0}^{2} \nu_{0}^{3} v_{1}\left(2 \delta_{0}^{2} \Lambda+\sigma^{2}\right)+\delta_{0}^{6}\left(\delta_{0}^{2} \Lambda\left(\pi^{2}-4 v_{0}^{2}\right)+\left(\pi^{2}-3 v_{0}^{2}\right) \sigma^{2}\right)
\end{aligned}
$$


Table 11 Exact meaning of $H \rightarrow 0$ and $H \rightarrow \infty$, for large values of $\gamma$, and $\Lambda=1.2$ and $\sigma^{2}=5$

\begin{tabular}{lllll}
\hline$\gamma$ & Free-free & \multicolumn{3}{l}{ Rigid-rigid } \\
\cline { 2 - 4 } & $H \rightarrow 0$ (LTE) & $H \rightarrow \infty$ (LTE) & $H \rightarrow 0$ (LTE) & $H \rightarrow \infty$ (LTE) \\
\hline 10 & 0.057 & 218.53 & 0.057 & 182.33 \\
20 & 0.051 & 110.53 & 0.043 & 94.46 \\
30 & 0.045 & 73.97 & 0.05 & 63.69 \\
40 & 0.024 & 55.58 & 0.038 & 48.03 \\
50 & 0.017 & 44.52 & 0.023 & 38.55 \\
60 & 0.014 & 37.13 & 0.017 & 32.20 \\
70 & 0.011 & 31.84 & 0.014 & 27.64 \\
80 & 0.009 & 27.87 & 0.012 & 24.21 \\
90 & 0.008 & 24.78 & 0.01 & 21.54 \\
100 & 0.007 & 22.31 & 0.009 & 19.4 \\
\hline
\end{tabular}

Table 12 Exact meaning of $\gamma \rightarrow \infty$ and $\gamma H \rightarrow \infty$, for large values of $H$, and $\Lambda=1.2$ and $\sigma^{2}=5$

\begin{tabular}{lllll}
\hline $\mathrm{H}$ & Free-free & \multicolumn{3}{l}{ Rigid-rigid } \\
\cline { 2 - 5 } & $\gamma \rightarrow \infty($ LTE $)$ & $\gamma H \rightarrow \infty($ LTE $)$ & $\gamma \rightarrow \infty($ LTE $)$ & $\gamma H \rightarrow \infty($ LTE) \\
\hline 10 & 239.06 & 2390.56 & 229.3 & 2292.98 \\
20 & 126.38 & 2527.56 & 127.3 & 2554.58 \\
30 & 88.37 & 2651.10 & 92.38 & 2771.38 \\
40 & 69.11 & 2764.07 & 73.97 & 2959.70 \\
50 & 57.37 & 2868.48 & 62.50 & 3124.88 \\
60 & 49.43 & 2965.78 & 54.59 & 3275.02 \\
70 & 43.68 & 3057.08 & 48.76 & 3412.51 \\
80 & 39.30 & 3143.21 & 44.25 & 3539.71 \\
90 & 35.84 & 3224.85 & 40.65 & 3658.36 \\
100 & 33.03 & 3302.55 & 37.7 & 3769.75 \\
\hline
\end{tabular}

The critical wave number $v_{c}$ is computed from the obtained values of $v_{0}, v_{1}$ and $v_{2}$ and using this value of $v_{c}$ we can obtain critical Rayleigh number from Eq. (28) for large $\mathrm{H}$.

Case 3: For large values of $\gamma$

For large values of $\gamma$, i. e., $\frac{1}{\gamma}<<1, R a_{n l}^{F F}$ takes the form,

$$
R a_{n l}^{F F}=\frac{\delta^{4}\left(\delta^{2} \Lambda+\sigma^{2}\right)}{v^{2}}+\frac{\delta^{4}\left(\delta^{2} \Lambda+\sigma^{2}\right)}{v^{2}} \frac{1}{\gamma}-\frac{\delta^{6}\left(\delta^{2} \Lambda+\sigma^{2}\right)}{H v^{2}} \frac{1}{\gamma^{2}}+\cdots,
$$

where $\delta^{2}=v^{2}+\pi^{2}$.

We minimize Eq. (36) with respect to $v$ and obtain the expression:

$$
\begin{aligned}
& H\left(4 \Lambda v^{6}+2 v^{4}\left(3 \pi^{2} \Lambda+\sigma^{2}\right)-2 \pi^{4}\left(\pi^{2} \Lambda+\sigma^{2}\right)\right) \\
& +H\left(4 \Lambda v^{6}+2 v^{4}\left(3 \pi^{2} \Lambda+\sigma^{2}\right)-2 \pi^{4}\left(\pi^{2} \Lambda+\sigma^{2}\right)\right) \frac{1}{\gamma}
\end{aligned}
$$




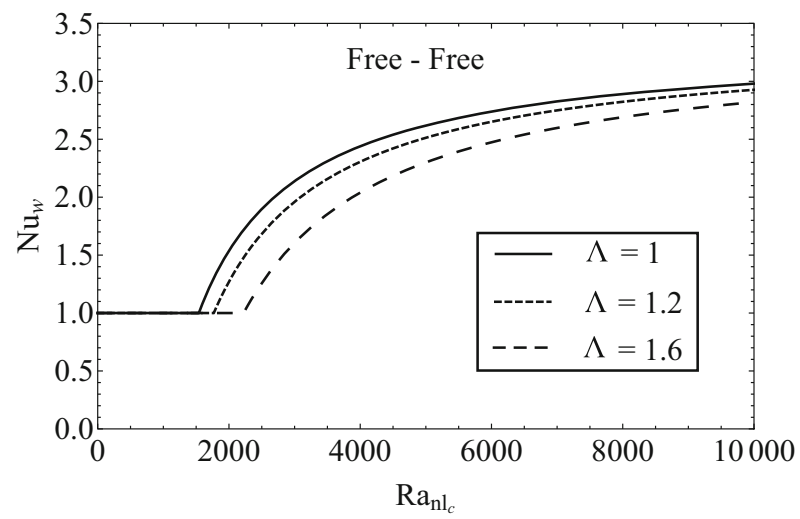

Fig. 10 Variation of $N u_{w}$ with $R a_{n l}$, for different values of $\Lambda$, for fixed values of $\sigma^{2}=5, \gamma=1$ and $\mathrm{H}=10$

$$
+2 \delta^{4}\left(\delta^{2} \Lambda\left(\pi^{2}-3 v^{2}\right)+\left(\pi^{2}-2 v^{2}\right) \sigma^{2}\right) \frac{1}{\gamma^{2}}=0
$$

We expand $v$ in the form,

$$
v=v_{0}+\frac{v_{1}}{\gamma}+\frac{v_{2}}{\gamma^{2}}+\cdots,
$$

where $v_{0}$ is the critical wave number for the LTE case given by the Eq. (21).

Substituting Eq. (38) in Eq. (36) and equating the coefficients of like powers of $\gamma$, we obtain the values of $v_{1}$ and $v_{2}$ as:

$$
\begin{aligned}
& v_{1}=\frac{\Delta_{7}^{\prime}}{\Delta_{8}^{\prime}}, \\
& v_{2}=\frac{\Delta_{9}^{\prime}}{H \Delta_{8}^{\prime}},
\end{aligned}
$$

where

$$
\begin{aligned}
\Delta_{7}^{\prime}= & \delta_{0}^{4} \Lambda\left(\pi^{2}-2 v_{0}^{2}\right)+\left(\pi^{4}-v_{0}^{4}\right) \sigma^{2}, \\
\Delta_{8}^{\prime}= & 4 v_{0}^{3}\left(3 \delta_{0}^{2} \Lambda+\sigma^{2}\right), \\
\Delta_{9}^{\prime}= & \delta_{0}^{4}\left(2 v_{0}^{2}\left(\pi^{2} \Lambda+\sigma^{2}\right)+3 \Lambda v_{0}^{4}-\pi^{2}\left(\pi^{2} \Lambda+\sigma^{2}\right)\right) \\
& -2 H v_{0}^{2} v_{1}\left(2 v_{0}\left(3 \Lambda v_{0}^{2}+3 \pi^{2} \Lambda+\sigma^{2}\right)+3 v_{1}\left(5 \Lambda v_{0}^{2}+3 \pi^{2} \Lambda+\sigma^{2}\right)\right) .
\end{aligned}
$$

The critical wave number $v_{c}$ is computed from the obtained values of $v_{0}, v_{1}$ and $v_{2}$ and using this value of $v_{c}$ we can obtain critical Rayleigh number from Eq. (36) for large $\gamma$.

Case 4: For large values of $\gamma H$

For large values of $\gamma H$, i. e. $\frac{1}{\gamma H}<<1, R a_{n l}^{F F}$ takes the form,

$$
R a_{n l}^{F F}=\frac{\delta^{4}\left(\delta^{2} \Lambda+\sigma^{2}\right)}{v^{2}}+\frac{\delta^{4} H\left(\delta^{2} \Lambda+\sigma^{2}\right)}{v^{2}} \frac{1}{\gamma H}-\frac{\delta^{6} H\left(\delta^{2} \Lambda+\sigma^{2}\right)}{v^{2}} \frac{1}{(\gamma H)^{2}}+\cdots,
$$

where $\delta^{2}=v^{2}+\pi^{2}$. 


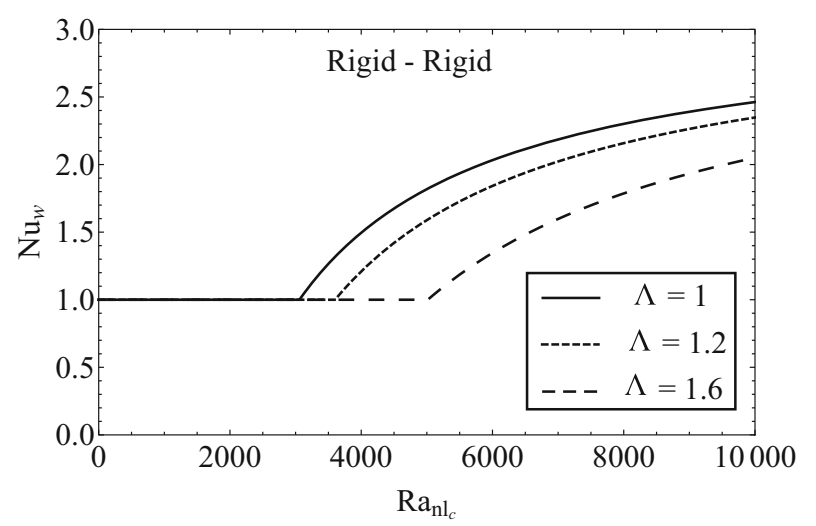

Fig. 11 Variation of $N u_{w}$ with $R a_{n l}$, for different values of $\Lambda$, for fixed values of $\sigma^{2}=5, \gamma=10$ and $\mathrm{H}=5$

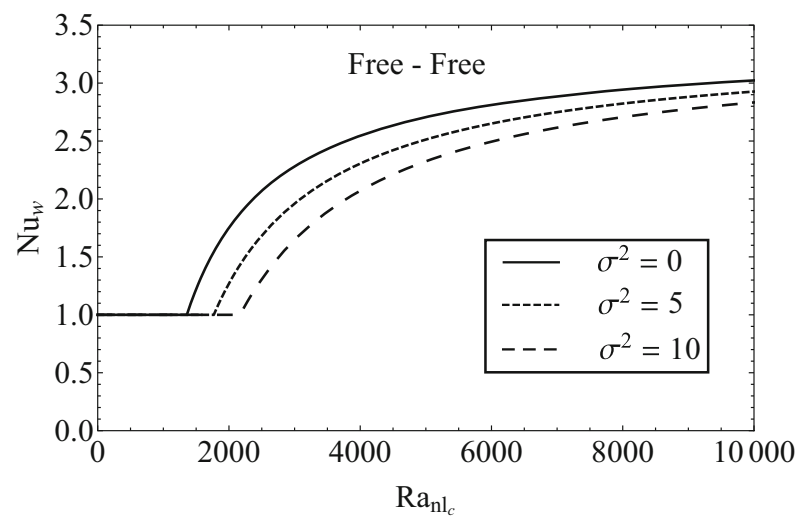

Fig. 12 Variation of $N u_{w}$ with $R a_{n l}$, for different values of $\sigma^{2}$, for fixed values of $\Lambda=1.2, \gamma=1$ and $\mathrm{H}=10$

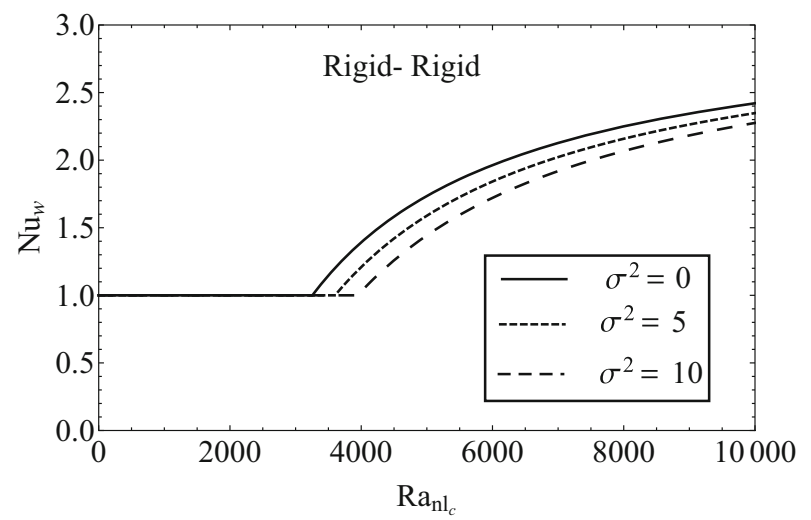

Fig. 13 Variation of $N u_{w}$ with $R a_{n l}$, for different values of $\sigma^{2}$, for fixed values of $\Lambda=1.2, \gamma=10$ and $\mathrm{H}=5$ 


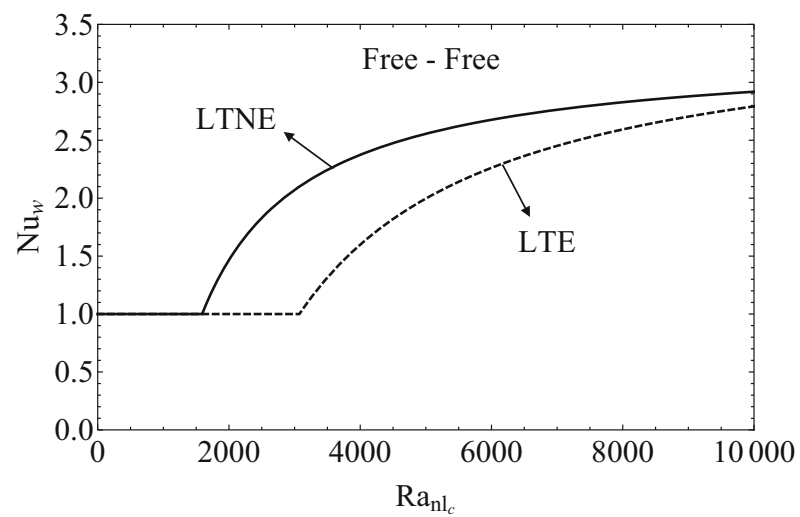

Fig. 14 Variation of $N u_{w}$ with $R a_{n l}$ of LTNE and LTE for $\Lambda=1.2, \sigma^{2}=10$ and $\gamma=1$

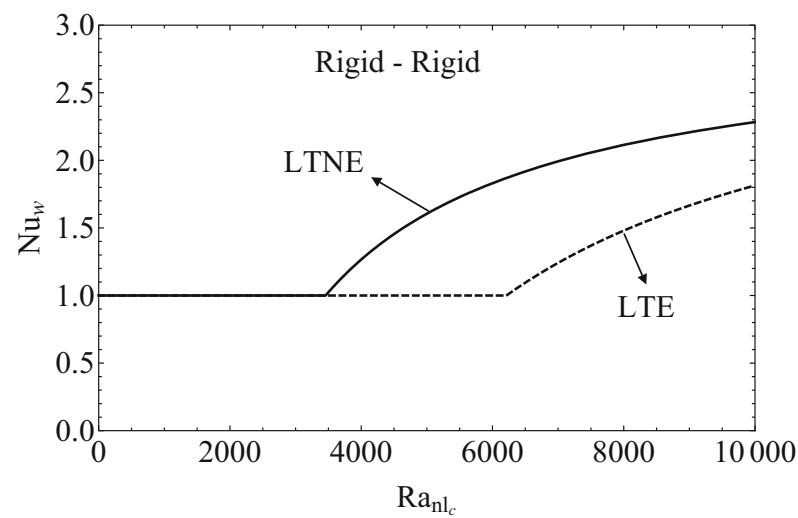

Fig. 15 Variation of $N u_{w}$ with $R a_{n l}$ of LTNE and LTE for $\Lambda=1.2, \sigma^{2}=10$ and $\gamma=1$

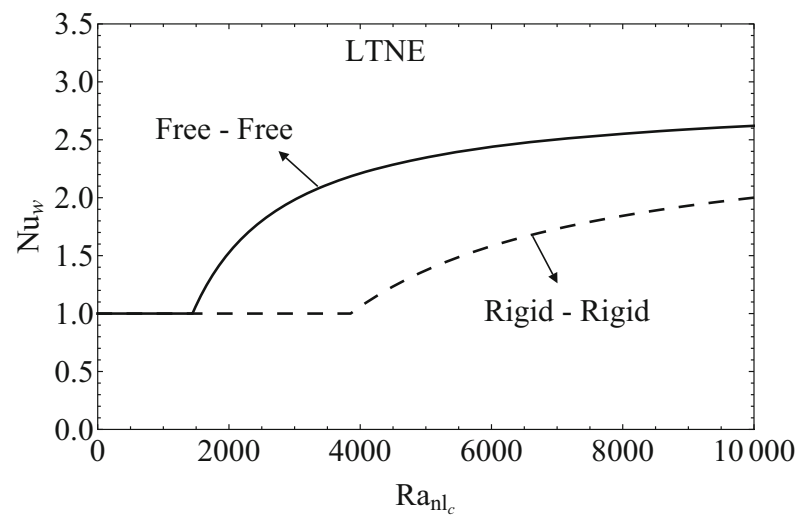

Fig. 16 Variation of $N u_{w}$ with $R a_{n l}$ of free-free and rigid-rigid boundaries, for $\Lambda=1.2, \sigma^{2}=5, \gamma=5$ and $\mathrm{H}=10$ 
We minimize Eq. (44) with respect to $v$ and obtain the expression:

$$
\begin{aligned}
& 4 \Lambda v^{6}+2 v^{4}\left(3 \pi^{2} \Lambda+\sigma^{2}\right)-2 \pi^{4}\left(\pi^{2} \Lambda+\sigma^{2}\right) \\
& +2 H\left(2 \Lambda v^{6}+v^{4}\left(3 \pi^{2} \Lambda+\sigma^{2}\right)-\pi^{4}\left(\pi^{2} \Lambda+\sigma^{2}\right)\right) \frac{1}{\gamma H} \\
& +2 \delta^{4} H\left(\delta^{2} \Lambda\left(\pi^{2}-3 v^{2}\right)+\left(\pi^{2}-2 v^{2}\right) \sigma^{2}\right) \frac{1}{(\gamma H)^{2}}=0
\end{aligned}
$$

We expand $v$ in the form,

$$
v=v_{0}+\frac{v_{1}}{\gamma H}+\frac{v_{2}}{(\gamma H)^{2}}+\cdots .
$$

where $v_{0}$ is given by Eq. (21).

Substituting Eq. (46) in Eq. (44) and equating the coefficients of like powers of $\gamma$, we obtain the values of $v_{1}$ and $v_{2}$ as:

$$
\begin{aligned}
& v_{1}=\frac{\Delta_{10}^{\prime}}{\Delta_{11}^{\prime}}, \\
& v_{2}=\frac{2 \Delta_{12}^{\prime}}{\Delta_{11}^{\prime}},
\end{aligned}
$$

where

$$
\begin{aligned}
\Delta_{10}^{\prime}= & H \delta_{0}^{4} \Lambda\left(\pi^{2}-2 v_{0}^{2}\right)+H\left(\pi^{4}-v_{0}^{4}\right) \sigma^{2}, \\
\Delta_{11}^{\prime}= & 4 v_{0}^{3}\left(3 \delta_{0}^{2} \Lambda+\sigma^{2}\right), \\
\Delta_{12}^{\prime}= & \delta_{0}^{2} H \Lambda\left(3 v_{0}^{6}+5 \pi^{2} v_{0}^{4}-12 v_{1} v_{0}^{3}+\pi^{4} v_{0}^{2}-\pi^{6}\right) \\
& +H\left(2 v_{0}^{6}+3 \pi^{2} v_{0}^{4}-4 v_{1} v_{0}^{3}-\pi^{6}\right) \sigma^{2}-6 v_{0}^{2} v_{1}^{2}\left(5 \Lambda v_{0}^{2}+3 \pi^{2} \Lambda+\sigma^{2}\right) .
\end{aligned}
$$

The critical wave number $v_{c}$ is computed from the obtained values of $v_{0}, v_{1}$ and $v_{2}$ and using this value of $v_{c}$, we can obtain critical Rayleigh number from Eq. (44) for large $\gamma H$.

\section{Rigid-Rigid, Isothermal Boundaries}

The boundary conditions suitable for rigid-rigid isothermal boundaries are:

$$
\Psi=\frac{\partial \Psi}{\partial Z}=\Theta_{n l}=\Theta_{s} \quad \text { at } Z= \pm \frac{1}{2} .
$$

The eigen solutions of the Eqs. (11)-(13) are:

$$
\left.\begin{array}{rl}
\Psi & =A \sin (v X) C_{f}(Z), \\
\Theta_{n l} & =B \cos (v X) \sin \left[\pi\left(Z+\frac{1}{2}\right)\right], \\
\Theta_{s} & =C \cos (v X) \sin \left[\pi\left(Z+\frac{1}{2}\right)\right],
\end{array}\right\},
$$

where

$$
C_{f}(Z)=\frac{\cosh \left(\mu_{1} Z\right)}{\cosh \left(\frac{\mu_{1}}{2}\right)}-\frac{\cos \left(\mu_{1} Z\right)}{\cos \left(\frac{\mu_{1}}{2}\right)}
$$


is the Chandrasekhar function [6,7,10] and $\mu_{1}=4.73004074$. Following the procedure used for free-free, isothermal boundaries, we can get the expression for the critical value of nanoliquid Rayleigh number for rigid-rigid, isothermal boundaries as:

$$
R a_{n l_{c}}^{R R}=\frac{\delta_{c}^{2} Q_{1}}{2 F_{3}^{2} \nu_{c}^{2}}\left(1+\frac{H}{\delta_{c}^{2}+\gamma H}\right),
$$

where

$$
\begin{aligned}
& \delta_{c}^{2}=v_{c}^{2}+\pi^{2}, \\
& Q_{1}=F_{1} \Lambda\left(v_{c}^{4}+\mu_{1}^{4}\right)+F_{1} v_{c}^{2} \sigma^{2}+F_{2} \mu_{1}^{2}\left(2 v_{c}^{2} \Lambda+\sigma^{2}\right), \\
& F_{1}=\frac{1}{1+\cos \mu_{1}}+\frac{1}{1+\cosh \mu_{1}}-\frac{\tan \left(\frac{\mu_{1}}{2}\right)}{\mu_{1}}-\frac{\tanh \left(\frac{\mu_{1}}{2}\right)}{\mu_{1}}, \\
& F_{2}=\frac{1}{1+\cos \mu_{1}}-\frac{1}{1+\cosh \mu_{1}}+\frac{\tan \left(\frac{\mu_{1}}{2}\right)}{\mu_{1}}-\frac{\tanh \left(\frac{\mu_{1}}{2}\right)}{\mu_{1}}, \\
& F_{3}=\frac{4 \pi \mu_{1}^{2}}{\pi^{4}-\mu_{1}^{4}} .
\end{aligned}
$$

\section{Asymptotic Analysis}

Case 1: For small values of $\mathrm{H}$

We expand Ra given by Eq. (54) in a power series in $\mathrm{H}$ as

$$
R a_{n l}^{R R}=\frac{\delta^{2} Q_{1}}{2 v^{2} F_{3}^{2}}+\frac{Q_{1}}{2 v^{2} Q_{3}} H-\frac{\gamma Q_{1}}{2 v^{2} \delta^{2} F_{3}^{2}} H^{2}+\cdots,
$$

where $Q_{1}$ and $F_{3}$ are given by Eqs. (56) and (59) respectively and $\delta^{2}=v^{2}+\pi^{2}$. Setting $\frac{\partial R a_{n l}^{R R}}{\partial v}=0$ for minimizing $R a_{n l}^{R R}$, we get:

$$
\begin{aligned}
& \delta^{4}\left(F_{2} \mu_{1}^{2}\left(2 \Lambda v^{4}-\pi^{2} \sigma^{2}\right)+F_{1}\left(v^{4}\left(2 \Lambda v^{2}+\pi^{2} \Lambda+\sigma^{2}\right)-\pi^{2} \Lambda \mu_{1}^{4}\right)\right) \\
& \quad+\delta^{4}\left(F_{1} \Lambda\left(v^{4}-\mu_{1}^{4}\right)-F_{2} \mu_{1}^{2} \sigma^{2}\right) H+\gamma\left(F_{2} \mu_{1}^{2}\left(2 \Lambda v^{4}+\left(2 v^{2}+\pi^{2}\right) \sigma^{2}\right)\right. \\
& \left.\quad+F_{1}\left(\Lambda \mu_{1}^{4}\left(2 v^{2}+\pi^{2}\right)+v^{4}\left(\sigma^{2}-\pi^{2} \Lambda\right)\right)\right) H^{2}=0 .
\end{aligned}
$$

We expand $v$ in terms of $\mathrm{H}$ as

$$
v=v_{0}+v_{1} H+v_{2} H^{2}+\cdots,
$$

where $v_{0}$ is the critical wave number for the LTE case given by,

$$
v_{0}=\sqrt{\frac{Q_{2}^{2}-Q_{2} Q_{3}+Q_{3}^{2}}{6 \Lambda F_{1} Q_{3}}}
$$

where

$$
\begin{aligned}
Q_{2}= & F_{1}\left(\pi^{2} \Lambda+\sigma^{2}\right)+2 F_{2} \Lambda \mu_{1}^{2}, \\
Q_{3}= & \left(F_{1}^{3}\left(54 \pi^{2} \Lambda^{3} \mu_{1}^{4}-\left(\pi^{2} \Lambda+\sigma^{2}\right)^{3}\right)-8 F_{2}^{3} \Lambda^{3} \mu_{1}^{6}-12 F_{1} F_{2}^{2} \Lambda^{2} \mu_{1}^{4}\right. \\
& \left(\pi^{2} \Lambda+\sigma^{2}\right)-6 F_{1}^{2} F_{2} \Lambda \mu_{1}^{2}\left(\pi^{4} \Lambda^{2}-7 \pi^{2} \Lambda \sigma^{2}+\sigma^{4}\right)+6 \sqrt{3} \pi\left(-F_{1}^{2} \Lambda^{2} \mu_{1}^{2}\right.
\end{aligned}
$$




$$
\begin{aligned}
& \left(F_{1} \Lambda \mu_{1}^{2}+F_{2} \sigma^{2}\right)\left(8 F_{2}^{3} \Lambda^{3} \mu_{1}^{6}+3 F_{1} \Lambda^{2} \mu_{1}^{4}\left(4 F_{2}^{2}\left(\pi^{2} \Lambda+\sigma^{2}\right)-9 \pi^{2} F_{1}^{2} \Lambda\right)\right. \\
& \left.\left.\left.+3 F_{1}^{2} F_{2} \Lambda \mu_{1}^{2}\left(2 \pi^{4} \Lambda^{2}-5 \pi^{2} \Lambda \sigma^{2}+2 \sigma^{4}\right)+F_{1}^{3}\left(\pi^{2} \Lambda+\sigma^{2}\right)^{3}\right)\right)^{1 / 2}\right)^{1 / 3}
\end{aligned}
$$

and $F_{1}$ is given by Eq. (57).

Substituting Eq. (62) in Eq. (61) and equating the coefficients of like powers of H, we obtain $v_{1}$ and $v_{2}$ as:

$$
\begin{aligned}
& v_{1}=-\frac{\delta_{0}^{2} \Delta_{1}}{\Delta_{2}}, \\
& v_{2}=\frac{\Delta_{3}}{\delta_{0}^{2} \Delta_{2}},
\end{aligned}
$$

where

$$
\begin{aligned}
\delta_{0}^{2}= & v_{0}^{2}+\pi^{2}, \\
\Delta_{1}= & F_{1} \Lambda\left(v_{0}^{4}-\mu_{1}^{4}\right)-F_{2} \mu_{1}^{2} \sigma^{2}, \\
\Delta_{2}= & 4 v_{0}\left(F_{2} \mu_{1}^{2}\left(4 \Lambda v_{0}^{4}+2 \pi^{2} \Lambda v_{0}^{2}-\pi^{2} \sigma^{2}\right)+F_{1}\left(v _ { 0 } ^ { 2 } \left(\Lambda\left(5 v_{0}^{4}+5 \pi^{2} v_{0}^{2}+\pi^{4}\right)\right.\right.\right. \\
& \left.\left.\left.+\left(2 v_{0}^{2}+\pi^{2}\right) \sigma^{2}\right)-\pi^{2} \Lambda \mu_{1}^{4}\right)\right), \\
\Delta_{3}= & F_{2} \mu_{1}^{2}\left(\sigma^{2}\left(-\left(\gamma\left(2 v_{0}^{2}+\pi^{2}\right)-2 v_{1}\left(2 v_{0}^{3}+\pi^{2}\left(3 v_{0} v_{1}+2\right) v_{0}+\pi^{4} v_{1}\right)\right)\right)\right. \\
& \left.-2 \Lambda v_{0}^{2}\left(\gamma v_{0}^{2}+2\left(14 v_{0}^{4}+15 \pi^{2} v_{0}^{2}+3 \pi^{4}\right) v_{1}^{2}\right)\right)-F_{1}\left(\Lambda \mu _ { 1 } ^ { 4 } \left(\gamma\left(2 v_{0}^{2}+\pi^{2}\right)\right.\right. \\
& \left.-2 v_{1}\left(2 v_{0}^{3}+\pi^{2}\left(3 v_{0} v_{1}+2\right) v_{0}+\pi^{4} v_{1}\right)\right)+v_{0}^{2}\left(\Lambda \left(2 v _ { 1 } \left(\left(45 v_{0} v_{1}+4\right) v_{0}^{5}\right.\right.\right. \\
& \left.\left.+2 \pi^{2}\left(35 v_{0} v_{1}+3\right) v_{0}^{3}+2 \pi^{4}\left(15 v_{0} v_{1}+1\right) v_{0}+3 \pi^{6} v_{1}\right)-\pi^{2} \gamma v_{0}^{2}\right) \\
& \left.\left.+\sigma^{2}\left(\gamma v_{0}^{2}+2\left(14 v_{0}^{4}+15 \pi^{2} v_{0}^{2}+3 \pi^{4}\right) v_{1}^{2}\right)\right)\right) .
\end{aligned}
$$

Using the obtained values of $v_{0}, v_{1}, v_{2}$ in Eq. (62) we get the critical wave number and using this in the Eq. (60) we can obtain the critical Rayleigh number for small $\mathrm{H}$.

Case 2: For large values of $\mathrm{H}$

For large values of $\mathrm{H}, R a_{n l}^{R R}$ takes the form

$$
R a_{n l}^{R R}=\frac{\delta^{2}(1+\gamma) Q_{1}}{2 v^{2} \gamma F_{3}^{2}}-\frac{\delta^{4} Q_{1}}{2 v^{2} \gamma^{2} F_{3}^{2}} \frac{1}{H}+\frac{\delta^{6} Q_{1}}{2 v^{2} \gamma^{3} F_{3}^{2}} \frac{1}{H^{2}}+\cdots,
$$

where $Q_{1}$ and $F_{3}$ are given by Eq. (56) and Eq. (59) respectively and $\delta^{2}=v^{2}+\pi^{2}$.

Setting $\frac{\partial R a_{n l}^{R R}}{\partial v}=0$, we get:

$$
\begin{aligned}
& \gamma^{2}(\gamma+1)\left(F_{2} \mu_{1}^{2}\left(2 \Lambda v^{4}-\pi^{2} \sigma^{2}\right)+F_{1}\left(v^{4}\left(2 \Lambda v^{2}+\pi^{2} \Lambda+\sigma^{2}\right)-\pi^{2} \Lambda \mu_{1}^{4}\right)\right) \\
& -\left(\gamma \delta ^ { 2 } \left(F_{2} \mu_{1}^{2}\left(4 \Lambda v^{4}+v^{2} \sigma^{2}-\pi^{2} \sigma^{2}\right)\right.\right. \\
& \left.\left.+F_{1}\left(\Lambda \mu_{1}^{4}(v-\pi)(v+\pi)+v^{4}\left(3 \Lambda v^{2}+\pi^{2} \Lambda+2 \sigma^{2}\right)\right)\right)\right) \frac{1}{H} \\
& +\left(\delta ^ { 4 } \left(F_{2} \mu_{1}^{2}\left(6 \Lambda v^{4}+\left(2 v^{2}-\pi^{2}\right) \sigma^{2}\right)+F_{1}\left(\Lambda \mu_{1}^{4}\left(2 v^{2}-\pi^{2}\right)\right.\right.\right. \\
& \left.\left.\left.\quad+v^{4}\left(4 \Lambda v^{2}+\pi^{2} \Lambda+3 \sigma^{2}\right)\right)\right)\right) \frac{1}{H^{2}}=0
\end{aligned}
$$


We expand $v$ in terms of $\frac{1}{H}<<1$ as

$$
v=v_{0}+\frac{v_{1}}{H}+\frac{v_{2}}{H^{2}}+\cdots,
$$

where $v_{0}$ is the critical wave number for the LTE case given by Eq. (63).

Substituting Eq. (74) in Eq. (73) and equating the coefficients of like powers of H, we obtain the values of $v_{1}$ and $v_{2}$ as:

$$
\begin{aligned}
& v_{1}=\frac{\delta_{0}^{2} \Delta_{4}}{\Delta_{5}}, \\
& v_{2}=\frac{\Delta_{6}}{\gamma \Delta_{5}}
\end{aligned}
$$

where

$$
\begin{aligned}
\Delta_{4}= & F_{1}\left(\Lambda \mu_{1}^{4}\left(v_{0}^{2}-\pi^{2}\right)+v_{0}^{4}\left(3 \Lambda v_{0}^{2}+\pi^{2} \Lambda+2 \sigma^{2}\right)\right) \\
& +F_{2} \mu_{1}^{2}\left(4 \Lambda v_{0}^{4}+v_{0}^{2} \sigma^{2}-\pi^{2} \sigma^{2}\right), \\
\Delta_{5}= & 4 \gamma(\gamma+1) v_{0}^{3}\left(2 F_{2} \Lambda \mu_{1}^{2}+F_{1}\left(3 \Lambda v_{0}^{2}+\pi^{2} \Lambda+\sigma^{2}\right)\right), \\
\Delta_{6}= & F_{2} \mu_{1}^{2}\left(v _ { 0 } ^ { 2 } \left(-12 \gamma^{2}(\gamma+1) \Lambda v_{1}^{2}+4 \gamma v_{1} v_{0}\left(6 \Lambda v_{0}^{2}+4 \pi^{2} \Lambda+\sigma^{2}\right)\right.\right. \\
& \left.\left.+v_{0}^{2}\left(-\left(6 \delta_{0}^{4} \Lambda+\left(2 v_{0}^{2}+3 \pi^{2}\right) \sigma^{2}\right)\right)\right)+\pi^{6} \sigma^{2}\right)+F_{1}\left(v _ { 0 } ^ { 2 } \left(2 \gamma v _ { 1 } \left(2 v_{0}\right.\right.\right. \\
& \left(3 v_{0}^{2}\left(2 \pi^{2} \Lambda+\sigma^{2}\right)+6 \Lambda v_{0}^{4}+\pi^{4} \Lambda+2 \pi^{2} \sigma^{2}\right)-3 \gamma(\gamma+1) v_{1} \\
& \left.\left.\left(5 \Lambda v_{0}^{2}+\pi^{2} \Lambda+\sigma^{2}\right)\right)-\delta_{0}^{4} v_{0}^{2}\left(4 \Lambda v_{0}^{2}+\pi^{2} \Lambda+3 \sigma^{2}\right)\right)+\Lambda \mu_{1}^{4}\left(4 \gamma v_{1} v_{0}^{3}\right. \\
& \left.\left.-2 v_{0}^{6}-3 \pi^{2} v_{0}^{4}+\pi^{6}\right)\right) .
\end{aligned}
$$

The critical wave number $v_{c}$ is computed from the obtained values of $v_{0}, v_{1}$ and $v_{2}$ and using this value of $v_{c}$ we can obtain critical Rayleigh number from Eq. (72) for large $\mathrm{H}$.

Case 3: For large values of $\gamma$

For large values of $\gamma$, i. e., $\frac{1}{\gamma}<<1, R a_{n l}^{R R}$ takes the form

$$
R a_{n l}^{R R}=\frac{\delta^{2} Q_{1}}{2 v^{2} F_{3}^{2}}+\frac{\delta^{2} Q_{1}}{2 v^{2} F_{3}^{2}} \frac{1}{\gamma}-\frac{\delta^{4} Q_{1}}{2 v^{2} H F_{3}^{2}} \frac{1}{\gamma^{2}}+\cdots,
$$

where $Q_{1}$ and $F_{3}$ are given by Eq. (56) and Eq. (59) respectively and $\delta^{2}=v^{2}+\pi^{2}$.

We minimize Eq. (80) with respect to $v$ and obtain the expression:

$$
\begin{aligned}
& H\left(F_{2} \mu_{1}^{2}\left(2 \Lambda v^{4}-\pi^{2} \sigma^{2}\right)+F_{1}\left(v^{4}\left(2 \Lambda v^{2}+\pi^{2} \Lambda+\sigma^{2}\right)-\pi^{2} \Lambda \mu_{1}^{4}\right)\right) \\
& +\left(H\left(F_{2} \mu_{1}^{2}\left(2 \Lambda v^{4}-\pi^{2} \sigma^{2}\right)+F_{1}\left(v^{4}\left(2 \Lambda v^{2}+\pi^{2} \Lambda+\sigma^{2}\right)-\pi^{2} \Lambda \mu_{1}^{4}\right)\right)\right) \frac{1}{\gamma} \\
& -\left(\delta ^ { 2 } \left(F_{2} \mu_{1}^{2}\left(4 \Lambda v^{4}+v^{2} \sigma^{2}-\pi^{2} \sigma^{2}\right)+F_{1}\left(\Lambda \mu_{1}^{4}\left(v^{2}-\pi^{2}\right)\right.\right.\right. \\
& \left.\left.\left.+v^{4}\left(3 \Lambda v^{2}+\pi^{2} \Lambda+2 \sigma^{2}\right)\right)\right)\right) \frac{1}{\gamma^{2}}=0 .
\end{aligned}
$$

We expand $v$ in the form,

$$
v=v_{0}+\frac{v_{1}}{\gamma}+\frac{v_{2}}{\gamma^{2}}+\cdots,
$$

where $v_{0}$ is the critical wave number for the LTE case given by Eq. (63). 
Substituting Eq. (82) in Eq. (80) and equating the coefficients of like powers of $\gamma$, we obtain the values of $v_{1}$ and $v_{2}$ as:

$$
\begin{aligned}
& v_{1}=\frac{\Delta_{7}}{\Delta_{8}}, \\
& v_{2}=\frac{\Delta_{9}}{H \Delta_{8}},
\end{aligned}
$$

where

$$
\begin{aligned}
\Delta_{7}= & F_{1}\left(\pi^{2} \Lambda \mu_{1}^{4}-v_{0}^{4}\left(2 \Lambda v_{0}^{2}+\pi^{2} \Lambda+\sigma^{2}\right)\right)+F_{2} \mu_{1}^{2}\left(\pi^{2} \sigma^{2}-2 \Lambda v_{0}^{4}\right), \\
\Delta_{8}= & 4 v_{0}^{3}\left(2 F_{2} \Lambda \mu_{1}^{2}+F_{1}\left(3 \Lambda v_{0}^{2}+\pi^{2} \Lambda+\sigma^{2}\right)\right), \\
\Delta_{9}= & F_{1}\left(v _ { 0 } ^ { 2 } \left(\delta_{0}^{2} v_{0}^{2}\left(3 \Lambda v_{0}^{2}+\pi^{2} \Lambda+2 \sigma^{2}\right)-4 H v_{1} v_{0}\left(3 \Lambda v_{0}^{2}+\pi^{2} \Lambda+\sigma^{2}\right)\right.\right. \\
& \left.\left.\quad-6 H v_{1}^{2}\left(5 \Lambda v_{0}^{2}+\pi^{2} \Lambda+\sigma^{2}\right)\right)+\Lambda \mu_{1}^{4}\left(v_{0}^{4}-\pi^{4}\right)\right)+F_{2} \mu_{1}^{2}\left(4 \Lambda v_{0}^{2}\right. \\
& \left.\left(-2 H v_{1} v_{0}-3 H v_{1}^{2}+v_{0}^{4}+\pi^{2} v_{0}^{2}\right)+\left(v_{0}^{4}-\pi^{4}\right) \sigma^{2}\right) .
\end{aligned}
$$

The critical wave number $v_{c}$ is computed from the obtained values of $v_{0}, v_{1}$ and $v_{2}$ and using this value of $v_{c}$ we can obtain critical Rayleigh number from Eq. (80) for large $\gamma$.

Case 4: For large values of $\gamma H$

For large values of $\gamma H$, i. e., $\frac{1}{\gamma H}<<1, R a_{n l}^{R R}$ takes the form

$$
R a_{n l}^{R R}=\frac{\delta^{2} Q_{1}}{2 v^{2} F_{3}^{2}}+\frac{\delta^{2} Q_{1} H}{2 v^{2} F_{3}^{2}} \frac{1}{\gamma H}-\frac{\delta^{4} H Q_{1}}{2 v^{2} F_{3}^{2}} \frac{1}{(\gamma H)^{2}}+\cdots,
$$

where $Q_{1}$ and $F_{3}$ are given by Eq. (56) and Eq. (59) respectively and $\delta^{2}=v^{2}+\pi^{2}$.

We minimize Eq. (88) with respect to $v$ and obtain the expression:

$$
\begin{aligned}
& F_{2} \mu_{1}^{2}\left(2 \Lambda v^{4}-\pi^{2} \sigma^{2}\right)+F_{1}\left(v^{4}\left(2 \Lambda v^{2}+\pi^{2} \Lambda+\sigma^{2}\right)-\pi^{2} \Lambda \mu_{1}^{4}\right) \\
& +\left(H\left(F_{2} \mu_{1}^{2}\left(2 \Lambda v^{4}-\pi^{2} \sigma^{2}\right)+F_{1}\left(v^{4}\left(2 \Lambda v^{2}+\pi^{2} \Lambda+\sigma^{2}\right)-\pi^{2} \Lambda \mu_{1}^{4}\right)\right)\right) \frac{1}{\gamma H} \\
& -\left(H ( v ^ { 2 } + \pi ^ { 2 } ) \left(F_{2} \mu_{1}^{2}\left(4 \Lambda v^{4}+v^{2} \sigma^{2}-\pi^{2} \sigma^{2}\right)\right.\right. \\
& \left.\left.\quad+F_{1}\left(\Lambda \mu_{1}^{4}(v-\pi)(v+\pi)+v^{4}\left(3 \Lambda v^{2}+\pi^{2} \Lambda+2 \sigma^{2}\right)\right)\right)\right) \frac{1}{(\gamma H)^{2}}=0 .
\end{aligned}
$$

We expand $v$ in the form,

$$
v=v_{0}+\frac{v_{1}}{\gamma H}+\frac{v_{2}}{(\gamma H)^{2}}+\cdots,
$$

where $v_{0}$ is the critical wave number for the LTE case given by Eq. (63).

Substituting Eq. (90) in Eq. (88) and equating the coefficients of like powers of $\gamma$, we obtain the values of $v_{1}$ and $v_{2}$ as:

$$
\begin{aligned}
& v_{1}=\frac{\Delta_{10}}{\Delta_{11}}, \\
& v_{2}=\frac{\Delta_{12}}{\Delta_{11}},
\end{aligned}
$$

where

$$
\Delta_{10}=H\left(F_{2} \mu_{1}^{2}\left(\pi^{2} \sigma^{2}-2 \Lambda v_{0}^{4}\right)+F_{1}\left(\pi^{2} \Lambda \mu_{1}^{4}-v_{0}^{4}\left(2 \Lambda v_{0}^{2}+\pi^{2} \Lambda+\sigma^{2}\right)\right)\right),
$$




$$
\begin{aligned}
\Delta_{11}= & 4 v_{0}^{3}\left(2 F_{2} \Lambda \mu_{1}^{2}+F_{1}\left(3 \Lambda v_{0}^{2}+\pi^{2} \Lambda+\sigma^{2}\right)\right), \\
\Delta_{12}= & F_{1}\left(v _ { 0 } ^ { 2 } \left(H v_{0}\left(\delta_{0}^{2} v_{0}\left(3 \Lambda v_{0}^{2}+\pi^{2} \Lambda+2 \sigma^{2}\right)-4 v_{1}\left(3 \Lambda v_{0}^{2}+\pi^{2} \Lambda+\sigma^{2}\right)\right)\right.\right. \\
& \left.\left.-6 v_{1}^{2}\left(5 \Lambda v_{0}^{2}+\pi^{2} \Lambda+\sigma^{2}\right)\right)+H \Lambda \mu_{1}^{4}\left(v_{0}^{4}-\pi^{4}\right)\right)+F_{2} \mu_{1}^{2}\left(4 \Lambda v_{0}^{2}\right. \\
& \left.\left(H v_{0}\left(v_{0}^{3}+\pi^{2} v_{0}-2 v_{1}\right)-3 v_{1}^{2}\right)+H\left(v_{0}^{4}-\pi^{4}\right) \sigma^{2}\right) .
\end{aligned}
$$

The critical wave number $v_{c}$ is computed from the obtained values of $v_{0}, v_{1}$ and $v_{2}$ and using this value of $v_{c}$ we can obtain critical Rayleigh number from Eq. (88) for large $\gamma H$.

The non-linear analysis will now be used to study heat transport.

\section{Weakly Non-linear Stability Analysis for Steady Convection in the Case of Free-Free/Rigid-Rigid, Isothermal Boundaries}

\section{Free-Free, Isothermal Boundaries}

The truncated representation for making a weakly non-linear analysis for free-free, isothermal boundaries is

$$
\begin{aligned}
\Psi & =A \sin \left(v_{c} X\right) \sin \left[\pi\left(Z+\frac{1}{2}\right)\right], \\
\Theta_{n l} & \left.=B_{1} \cos \left(v_{c} X\right) \sin \left[\pi\left(Z+\frac{1}{2}\right)\right]+B_{2} \sin \left[2 \pi\left(Z+\frac{1}{2}\right)\right],\right\} . \\
\Theta_{s} & =C_{1} \cos \left(v_{c} X\right) \sin \left[\pi\left(Z+\frac{1}{2}\right)\right]+C_{2} \sin \left[2 \pi\left(Z+\frac{1}{2}\right)\right] .
\end{aligned}
$$

Substituting Eq. (96) into Eqs. (11)-(13) and using the orthogonality condition with the eigen functions on the resulting equations, we get the following algebraic equations:

$$
\begin{aligned}
a_{1} B_{1} k R a_{n l}+A \delta_{c}^{2}\left(\Lambda \delta_{c}^{2}+\sigma^{2}\right) & =0, \\
\frac{\left(1+\pi B_{2}\right) v_{c}}{a_{1}\left(H+\delta_{c}^{2}\right)} A+B_{1}-\frac{H}{H+\delta_{c}^{2}} C_{1} & =0, \\
\mathrm{AB}_{1}-\frac{2 a_{1}\left(H+4 \pi^{2}\right)}{\pi v_{c}} B_{2}+\frac{2 a_{1} H}{\pi v_{c}} C_{2} & =0, \\
B_{1}-\frac{\left(\delta_{c}^{2}+\gamma H\right)}{\gamma H} C_{1} & =0, \\
B_{2}-\frac{\left(H+4 \pi^{2}\right)}{\gamma H} C_{2} & =0,
\end{aligned}
$$

Solving Eqs. (97)-(101), we get

$$
\begin{aligned}
& A^{2}=\frac{8 a_{1}^{2} \delta_{c}^{2}\left(H+4 \pi^{2}+\gamma H\right)\left(H+\delta_{c}^{2}+\gamma H\right) r}{v_{c}^{2}\left(\gamma H+4 \pi^{2}\right)\left(\delta_{c}^{2}+\gamma H\right)}\left[1-\frac{1}{r}\right], \\
& B_{1}=-\frac{v_{c}\left(\delta_{c}^{2}+\gamma H\right)}{a_{1} \delta_{c}^{2}\left(H+\delta_{c}^{2}+\gamma H\right) r} A, \\
& B_{2}=-\frac{v_{c}^{2}\left(\gamma H+4 \pi^{2}\right)\left(\delta_{c}^{2}+\gamma H\right)}{8 \pi a_{1}^{2} \delta_{c}^{2}\left(H+4 \pi^{2}+\gamma H\right)\left(H+\delta_{c}^{2}+\gamma H\right) r} A^{2}=-\frac{1}{\pi}\left[1-\frac{1}{r}\right],
\end{aligned}
$$




$$
\begin{aligned}
C_{1} & =-\frac{\gamma H v_{c}}{a_{1} r \delta_{c}^{2}\left(\delta_{c}^{2}+\gamma H+H\right)} A, \\
C_{2} & =-\frac{\gamma H v_{c}^{2}\left(\delta_{c}^{2}+\gamma H\right)}{8 \pi a_{1}^{2} \delta_{c}^{2}\left(H+4 \pi^{2}+\gamma H\right)\left(H+\delta_{c}^{2}+\gamma H\right) r} A^{2} \\
& =-\frac{\gamma H}{\pi\left(\gamma H+4 \pi^{2}\right)}\left[1-\frac{1}{r}\right],
\end{aligned}
$$

where

$$
r=\frac{R a_{n l}}{R a_{n l_{c}}^{F F}}
$$

is the scaled Rayleigh number.

\section{Rigid-Rigid, Isothermal Boundaries}

The truncated representation for making a steady, finite amplitude analysis for rigid-rigid, isothermal boundaries is

$$
\left.\begin{array}{rl}
\Psi & =A \sin \left(v_{c} X\right) C_{f}(Z), \\
\Theta_{n l} & =B_{1} \cos \left(v_{c} X\right) \sin \left[\pi\left(Z+\frac{1}{2}\right)\right]+B_{2} \sin \left[2 \pi\left(Z+\frac{1}{2}\right)\right], \\
\Theta_{s} & =C_{1} \cos \left(v_{c} X\right) \sin \left[\pi\left(Z+\frac{1}{2}\right)\right]+C_{2} \sin \left[2 \pi\left(Z+\frac{1}{2}\right)\right] .
\end{array}\right\} .
$$

Substituting Eq. (108) into Eqs. (11)-(13) and using orthogonalization condition with the eigen functions on the resulting equations, we get the following algebraic equations:

$$
\begin{aligned}
& v_{c}\left(F_{1}\left(\frac{\Lambda \mu_{1}^{4}}{v_{c}}+\sigma v_{c}+v_{c}^{3} \Lambda\right)+F_{2}\left(2 \Lambda \mu_{1}^{2} v_{c}+\frac{\mu_{1}^{2} \sigma^{2}}{v_{c}}\right)\right) A \\
& -a_{1} F_{3} v_{c} \mathrm{Ra}_{\mathrm{nl}} B_{1}=0 \\
& \frac{\left(2 F_{3}+F_{4} B_{2}\right) v_{c}}{a_{1}\left(H+\delta_{c}^{2}\right)} A-B_{1}+\frac{H}{H+\delta_{c}^{2}} C_{1}=0 \\
& \mathrm{AB}_{1}+\frac{2 a_{1}\left(H+4 \pi^{2}\right)}{v_{c} F_{4}} B_{2}-\frac{2 a_{1} H}{v_{c} F_{4}} C_{2}=0 \\
& B_{1}-\frac{\left(\delta_{c}^{2}+\gamma H\right)}{\gamma H} C_{1}=0 \\
& B_{2}-\frac{\left(H+4 \pi^{2}\right)}{\gamma H} C_{2}=0
\end{aligned}
$$

where $F_{1}, F_{2}, F_{3}$ are given by Eqs. (57)-(59) and

$$
F_{4}=\frac{16 \pi^{2} \mu_{1}^{2}\left(39 \pi^{4}+\mu_{1}^{4}\right)}{81 \pi^{8}-82 \pi^{4} \mu_{1}^{4}+\mu_{1}^{8}}
$$

Solving Eqs. (109)-(113), we get

$$
A^{2}=\frac{8 \pi^{2} a_{1}^{2} \delta_{c}^{2}\left(H+4 \pi^{2}+\gamma H\right)\left(H+\delta_{c}^{2}+\gamma H\right) r}{F_{4}^{2} v_{c}^{2}\left(\gamma H+4 \pi^{2}\right)\left(\delta_{c}^{2}+\gamma H\right)}\left[1-\frac{1}{r}\right],
$$




$$
\begin{aligned}
B_{1} & =\frac{2 F_{3} v_{c}\left(\delta_{c}^{2}+\gamma H\right)}{a_{1} \delta_{c}^{2}\left(H+\delta_{c}^{2}+\gamma H\right) r} A, \\
B_{2} & =-\frac{F_{3} F_{4} v_{c}^{2}\left(\gamma H+4 \pi^{2}\right)\left(\delta_{c}^{2}+\gamma H\right)}{4 \pi^{2} a_{1}^{2} r \delta_{c}^{2}\left(H+4 \pi^{2}+\gamma H\right)\left(H+\delta_{c}^{2}+\gamma H\right)} A^{2}=-\frac{2 F_{3}}{F_{4}}\left[1-\frac{1}{r}\right], \\
C_{1} & =\frac{2 \gamma F_{3} H v_{c}}{a_{1} \delta_{c}^{2}\left(H+\delta_{c}^{2}+\gamma H\right) r} A, \\
C_{2} & =-\frac{\gamma F_{3} F_{4} H v_{c}^{2}\left(\delta_{c}^{2}+\gamma H\right)}{4 \pi^{2} a_{1}^{2} r \delta_{c}^{2}\left(H+4 \pi^{2}+\gamma H\right)\left(H+\delta_{c}^{2}+\gamma H\right)} A^{2} \\
& =-\frac{2 F_{3} \gamma H}{F_{4}\left(\gamma H+4 \pi^{2}\right)}\left[1-\frac{1}{r}\right]
\end{aligned}
$$

where

$$
r=\frac{R a_{n l}}{R a_{n l_{c}}^{R R}}
$$

is the scaled Rayleigh number.

We next study heat transport in terms of the Nusselt number.

\section{Nusselt Number}

\section{Free-Free, Isothermal Boundaries}

The amount of heat transport by Rayleigh-Bénard convection for free-free, isothermal boundaries can be quantified in terms of the Nusselt number, $N u$, as follows:

$$
N u=\frac{\text { Heat transport by (conduction }+ \text { convection) }}{\text { Heat transport by conduction }} \text {. }
$$

Using Fourier first law and further simplyfying, we may write the expression for the Nusselt number for the nanoliquid and solid phases as

$$
\begin{gathered}
N u_{n l}=1+\frac{\left[-k_{n l} \int_{0}^{\frac{2 \pi}{v}} \frac{\partial \Theta_{n l}}{\partial Z} d X\right]_{Z=-\frac{1}{2}}}{\left[-k_{n l} \int_{0}^{\frac{2 \pi}{v}} \frac{d \Theta_{n l_{b}}}{d Z} d X\right]_{Z=-\frac{1}{2}}}, \\
N u_{s}=1+\frac{\left[-k_{s} \int_{0}^{\frac{2 \pi}{v}} \frac{\partial \Theta_{s}}{\partial Z} d X\right]_{Z=-\frac{1}{2}}}{\left[-k_{s} \int_{0}^{\frac{2 \pi}{v}} \frac{d \Theta_{s b}}{b Z} d X\right]_{Z=-\frac{1}{2}}},
\end{gathered}
$$

where $N u_{n l}$ is Nusselt number of the nanoliquid phase and $N u_{s}$ is that of the solid phase, $\Theta_{n l b}=\frac{T_{n l b}}{\Delta T}$ and $\Theta_{s b}=\frac{T_{s b}}{\Delta T}$. 
The weighted-average Nusselt number, $N u_{w}$, is given by

$$
N u_{w}=\phi N u_{n l}+(1-\phi) N u_{s} .
$$

Substituting Eqs. (7)-(8) and Eq. (96) in Eqs. (121) and (122) and completing the integration, we get

$$
\begin{aligned}
N u_{n l} & =1-2 \pi B_{2}, \\
N u_{s} & =1-2 \pi C_{2} .
\end{aligned}
$$

Substituting Eq. (104) and Eq. (106) in Eqs. (124) and (125), we get

$$
\begin{aligned}
& N u_{n l}^{F F}=1+2\left[1-\frac{1}{r}\right], \\
& N u_{s}^{F F}=1+2\left(\frac{\gamma H}{4 \pi^{2}+\gamma H}\right)\left[1-\frac{1}{r}\right] .
\end{aligned}
$$

Substituting Eqs. (126) and (127) in Eq. (123), we get

$$
N u_{w}^{F F}=1+2\left(\frac{4 \pi^{2} \phi+\gamma H}{4 \pi^{2}+\gamma H}\right)\left[1-\frac{1}{r}\right] .
$$

where $r$ is given by Eq. (107).

\section{Asymptotic Analysis for Small Values of H}

For small values of $\mathrm{H}$ with $R a_{n l}^{F F}$ given by Eq. (17) and $v$ given by Eq. (20), $N u_{w}^{F F}$ takes the form:

$$
N u_{w}^{F F}=G_{1}+G_{2} H+G_{3} H^{2}+\cdots,
$$

where

$$
\begin{aligned}
G_{1}= & 1+2 \phi\left(1-\frac{\delta_{0}^{2}\left(\delta_{0}^{2} \Lambda+\sigma^{2}\right)}{v_{0}^{2} R a_{n l}}\right) \\
G_{2}= & \frac{1}{2 \pi^{2} v_{0}^{3} R a_{n l}}\left(v_{0}^{5}\left(\pi^{2} \Lambda(3 \gamma(\phi-1)-4 \phi)+\gamma \sigma^{2}(\phi-1)\right)+\pi^{4} \nu_{0}(\gamma(\phi-1)\right. \\
& -4 \phi)\left(\pi^{2} \Lambda+\sigma^{2}\right)+\gamma \Lambda v_{0}^{7}(\phi-1)-8 \pi^{2} v_{1} v_{0}^{4} \phi\left(3 \pi^{2} \Lambda+\sigma^{2}\right)+8 \pi^{6} v_{1} \phi \\
& \left(\pi^{2} \Lambda+\sigma^{2}\right)-16 \pi^{2} \Lambda v_{1} v_{0}^{6} \phi-v_{0}^{3}\left(\pi ^ { 2 } \left(\pi^{2} \Lambda(-3 \gamma \phi+3 \gamma+8 \phi)\right.\right. \\
& \left.\left.\left.+2 \sigma^{2}(-\gamma \phi+\gamma+2 \phi)\right)+\gamma R a_{n l}(\phi-1)\right)\right), \\
G_{3}= & \frac{1}{8 \pi^{4} v_{0}^{4} R a_{n l}}\left(v_{0}^{6}\left(-\gamma^{2} \sigma^{2}(\phi-1)-\pi^{2} \gamma(3 \gamma-4) \Lambda(\phi-1)-96 \pi^{4} \Lambda v_{1}^{2} \phi\right)\right. \\
& -\gamma^{2} \Lambda v_{0}^{8}(\phi-1)-8 \pi^{2} v_{0}^{5}\left(v_{1}\left(\pi^{2} \Lambda(-3 \gamma \phi+3 \gamma+4 \phi)-\gamma \sigma^{2}(\phi-1)\right)\right. \\
& \left.+4 \pi^{2} v_{2} \phi\left(3 \pi^{2} \Lambda+\sigma^{2}\right)\right)-\pi^{4} \gamma v_{0}^{2}(\gamma(\phi-1)-20 \phi+4)\left(\pi^{2} \Lambda+\sigma^{2}\right) \\
& +8 \pi^{6} v_{0}\left(\pi^{2} \Lambda+\sigma^{2}\right)\left(v_{1}(\gamma(-\phi)+\gamma+4 \phi)+4 \pi^{2} \nu_{2} \phi\right) \\
& +16 \pi^{2} \Lambda v_{0}^{7}\left(\gamma v_{1}(\phi-1)-4 \pi^{2} v_{2} \phi\right)-48 \pi^{8} v_{1}^{2} \phi\left(\pi^{2} \Lambda+\sigma^{2}\right) \\
& +v_{0}^{4}\left(\gamma^{2} R a_{n l}(\phi-1)-\pi^{2}\left(\pi^{2}\left(3 \gamma^{2} \Lambda(\phi-1)+8 \gamma \Lambda(1-3 \phi)+16 v_{1}^{2} \sigma^{2} \phi\right)\right.\right. \\
& \left.\left.\left.+2(\gamma-2) \gamma \sigma^{2}(\phi-1)+48 \pi^{4} \Lambda v_{1}^{2} \phi\right)\right)\right) .
\end{aligned}
$$




\section{Asymptotic Analysis for Large Values of H, i. e., $\frac{1}{H} \ll 1$}

For large values of $\mathrm{H}$ with $R a_{n l}^{F F}$ given by Eq. (28) and $v$ given by Eq. (30), $N u_{w}^{F F}$ takes the form:

$$
N u_{w}^{F F}=G_{4}+G_{5} \frac{1}{H}+G_{6} \frac{1}{H^{2}}+\cdots,
$$

where

$$
\begin{aligned}
G_{4}= & -\frac{2(\gamma+1) \delta_{0}^{4}\left(\delta_{0}^{2} \Lambda+\sigma^{2}\right)}{\gamma v_{0}^{2} R a_{n l}}, \\
G_{5}= & \frac{1}{\gamma^{2} v_{0}^{3} R a_{n l}}\left(2 \left(v_{0}^{7}\left(\sigma^{2}-4 \pi^{2} \Lambda(\gamma(\phi-1)+\phi-2)\right)+\pi^{2} v_{0}^{5}\left(\sigma^{2}\right.\right.\right. \\
& \left.(-4(\gamma+1) \phi+4 \gamma+7)-6 \pi^{2} \Lambda(2(\gamma+1) \phi-2 \gamma-3)\right)-\pi^{6} v_{0}(4 \gamma(\phi-1) \\
& +4 \phi-5)\left(\pi^{2} \Lambda+\sigma^{2}\right)-2 \gamma(\gamma+1) v_{1} v_{0}^{4}\left(3 \pi^{2} \Lambda+\sigma^{2}\right)+2 \pi^{4} \gamma(\gamma+1) v_{1} \\
& \left(\pi^{2} \Lambda+\sigma^{2}\right)-4 \gamma(\gamma+1) \Lambda v_{1} v_{0}^{6}+\Lambda v_{0}^{9}+\pi^{2} v_{0}^{3}\left(\pi^{2}(4(\gamma-(\gamma+1) \phi)\right. \\
& \left.\left.\left.\left.\left(3 \pi^{2} \Lambda+2 \sigma^{2}\right)+11 \sigma^{2}\right)+16 \pi^{4} \Lambda+4 \gamma R a_{n l}(\phi-1)\right)\right)\right) \\
G_{6}= & -\frac{1}{\gamma^{3} v_{0}^{4} R a_{n l}}\left(2 \left(2 v _ { 0 } ^ { 6 } \left(3 \gamma^{2}(\gamma+1) \Lambda v_{1}^{2}+\pi^{6} \Lambda(-12(2 \gamma+3) \phi\right.\right.\right. \\
& \left.+24 \gamma+41)+\pi^{4} \sigma^{2}(-2(4 \gamma+7) \phi+8 \gamma+17)\right)+3 \pi^{4} \gamma^{2}(\gamma+1) v_{1}^{2} \\
& \left(\pi^{2} \Lambda+\sigma^{2}\right)+2 \pi^{2} v_{0}^{8}\left(\pi^{2} \Lambda(-8(\gamma+2) \phi+8 \gamma+21)-2 \sigma^{2}(\phi-2)\right) \\
& +4 \gamma v_{0}^{7}\left(v_{1}\left(4 \pi^{2} \Lambda(\gamma(\phi-1)+\phi-2)-\sigma^{2}\right)+\gamma(\gamma+1) \Lambda v_{2}\right)+2 \gamma v_{0}^{5} \\
& \left(\pi^{2} v_{1}\left(6 \pi^{2} \Lambda(2(\gamma+1) \phi-2 \gamma-3)+\sigma^{2}(4(\gamma+1) \phi-4 \gamma-7)\right)\right. \\
& \left.+\gamma(\gamma+1) v_{2}\left(3 \pi^{2} \Lambda+\sigma^{2}\right)\right)-\pi^{8} v_{0}^{2}(16 \gamma(\phi-1)+20 \phi-21)\left(\pi^{2} \Lambda+\sigma^{2}\right) \\
& -2 \pi^{4} \gamma v_{0}\left(\pi^{2} \Lambda+\sigma^{2}\right)\left(\pi^{2} v_{1}(4(\gamma+1) \phi-4 \gamma-5)+\gamma(\gamma+1) v_{2}\right) \\
& -6 \gamma \Lambda v_{1} v_{0}^{9}+v_{0}^{10}\left(\pi^{2} \Lambda(9-4 \phi)+\sigma^{2}\right)+\Lambda v_{0}^{12}+v_{0}^{4}\left(3 \pi^{2} \gamma^{2}(\gamma+1) \Lambda v_{1}^{2}\right. \\
& +\gamma^{2}(\gamma+1) v_{1}^{2} \sigma^{2}+\pi^{8} \Lambda(-48 \gamma(\phi-1)-64 \phi+69)+4 \pi^{6} \sigma^{2}(-8 \gamma \phi+8 \gamma \\
& \left.\left.\left.-11 \phi+12)+16 \pi^{4} \gamma R a_{n l}(\phi-1)\right)\right)\right)
\end{aligned}
$$

\section{Asymptotic Analysis for Large Values of $\gamma$, i. e., $\frac{1}{\gamma}<1$}

For large values of $\gamma$ with $R a_{n l}^{F F}$ given by Eq. (36) and $v$ given by Eq. (38), $N u_{w}^{F F}$ takes the form:

$$
N u_{w}^{F F}=G_{7}+G_{8} \frac{1}{\gamma}+G_{9} \frac{1}{\gamma^{2}}+\cdots,
$$

where

$$
\begin{aligned}
G_{7}=3 & -\frac{2 \delta_{0}^{4}\left(\delta_{0}^{2} \Lambda+\sigma^{2}\right)}{v_{0}^{2} R a_{n l}}, \\
G_{8}= & -\frac{1}{H v_{0}^{3} R a_{n l}}\left(2 \left(v_{0}^{5}\left(H+4 \pi^{2}(\phi-1)\right)\left(3 \pi^{2} \Lambda+\sigma^{2}\right)+\pi^{4} v_{0}(H\right.\right. \\
& \left.+4 \pi^{2}(\phi-1)\right)\left(\pi^{2} \Lambda+\sigma^{2}\right)+2 H v_{1} v_{0}^{4}\left(3 \pi^{2} \Lambda+\sigma^{2}\right)-2 \pi^{4} H v_{1}
\end{aligned}
$$




$$
\begin{aligned}
& \left(\pi^{2} \Lambda+\sigma^{2}\right)+\Lambda v_{0}^{7}\left(H+4 \pi^{2}(\phi-1)\right)+4 H \Lambda v_{1} v_{0}^{6}+\pi^{2} v_{0}^{3}((H \\
& \left.\left.\left.\left.+4 \pi^{2}(\phi-1)\right)\left(3 \pi^{2} \Lambda+2 \sigma^{2}\right)-4 R a_{n l}(\phi-1)\right)\right)\right) \\
G_{9}= & \frac{1}{H^{2} v_{0}^{4} R a_{n l}}\left(2 \left(v _ { 0 } ^ { 6 } \left(-6 H^{2} \Lambda v_{1}^{2}+\pi^{2} H\left(6 \pi^{2} \Lambda(3-2 \phi)+\sigma^{2}(7-4 \phi)\right)\right.\right.\right. \\
& \left.+16 \pi^{4}(\phi-1)\left(3 \pi^{2} \Lambda+\sigma^{2}\right)\right)-3 \pi^{4} H^{2} v_{1}^{2}\left(\pi^{2} \Lambda+\sigma^{2}\right)+v_{0}^{4}\left(-3 \pi^{2} H^{2} \Lambda v_{1}^{2}\right. \\
& -H^{2} v_{1}^{2} \sigma^{2}-4 \pi^{6}\left(H \Lambda(3 \phi-4)-8 \sigma^{2}(\phi-1)\right)+\pi^{4}\left(H \sigma^{2}(11-8 \phi)\right. \\
& \left.\left.-16 R a_{n l}(\phi-1)\right)+48 \pi^{8} \Lambda(\phi-1)\right)+v_{0}^{8}\left(H\left(\sigma^{2}-4 \pi^{2} \Lambda(\phi-2)\right)\right. \\
& \left.+16 \pi^{4} \Lambda(\phi-1)\right)-2 H v_{0}^{5}\left(3 \pi^{2} \Lambda+\sigma^{2}\right)\left(H\left(v_{1}+v_{2}\right)+4 \pi^{2} v_{1}(\phi-1)\right) \\
& +\pi^{6} v_{0}^{2}\left(H(5-4 \phi)+16 \pi^{2}(\phi-1)\right)\left(\pi^{2} \Lambda+\sigma^{2}\right)+2 \pi^{4} H v_{0}\left(\pi^{2} \Lambda+\sigma^{2}\right) \\
& \left(H\left(v_{1}+v_{2}\right)+4 \pi^{2} v_{1}(\phi-1)\right)-4 H \Lambda v_{0}^{7}\left(H\left(v_{1}+v_{2}\right)+4 \pi^{2} v_{1}(\phi-1)\right) \\
& \left.\left.+H \Lambda v_{0}^{10}\right)\right) .
\end{aligned}
$$

\section{Asymptotic Analysis for Large Values of $\gamma H$, i. e., $\frac{1}{\gamma H}<<1$}

For large values of $\gamma H$ with $R a_{n l}^{F F}$ given by Eq. (44) and $v$ given by Eq. (46), $N u_{w}^{F F}$ takes the form:

$$
N u_{w}^{F F}=G_{10}+G_{11} \frac{1}{\gamma H}+G_{12} \frac{1}{(\gamma H)^{2}}+\cdots,
$$

where $\delta_{0}^{2}=v_{0}^{2}+\pi^{2}$,

$$
\begin{aligned}
& G_{10}=3-\frac{2 \delta_{0}^{4}\left(\delta_{0}^{2} \Lambda+\sigma^{2}\right)}{v_{0}^{2} R a_{n l}}, \\
& G_{11}=-\frac{1}{v_{0}^{3} R a_{n l}}\left(2 \left(v_{0}^{5}\left(H+4 \pi^{2}(\phi-1)\right)\left(3 \pi^{2} \Lambda+\sigma^{2}\right)+\pi^{4} v_{0}(H\right.\right. \\
& \left.+4 \pi^{2}(\phi-1)\right)\left(\pi^{2} \Lambda+\sigma^{2}\right)+\Lambda v_{0}^{7}\left(H+4 \pi^{2}(\phi-1)\right)+\pi^{2} v_{0}^{3}((H \\
& \left.\left.+4 \pi^{2}(\phi-1)\right)\left(3 \pi^{2} \Lambda+2 \sigma^{2}\right)-4 R a_{n l}(\phi-1)\right)+2 v_{1} v_{0}^{4}\left(3 \pi^{2} \Lambda+\sigma^{2}\right) \\
& \left.\left.-2 \pi^{4} v_{1}\left(\pi^{2} \Lambda+\sigma^{2}\right)+4 \Lambda v_{1} v_{0}^{6}\right)\right) \text {, } \\
& G_{12}=\frac{1}{v_{0}^{4} R a_{n l}}\left(2 \left(v_{0}^{8}\left(H\left(\sigma^{2}-4 \pi^{2} \Lambda(\phi-2)\right)+16 \pi^{4} \Lambda(\phi-1)\right)+v_{0}^{6}\left(\pi^{2}\right.\right.\right. \\
& \left(H\left(6 \pi^{2} \Lambda(3-2 \phi)+\sigma^{2}(7-4 \phi)\right)+16 \pi^{2}(\phi-1)\left(3 \pi^{2} \Lambda+\sigma^{2}\right)\right) \\
& \left.-6 \Lambda v_{1}^{2}\right)-2 v_{0}^{5}\left(3 \pi^{2} \Lambda+\sigma^{2}\right)\left(H v_{1}+4 \pi^{2} v_{1}(\phi-1)+v_{2}\right)+\pi^{6} v_{0}^{2}(H \\
& \left.(5-4 \phi)+16 \pi^{2}(\phi-1)\right)\left(\pi^{2} \Lambda+\sigma^{2}\right)+2 \pi^{4} \nu_{0}\left(\pi^{2} \Lambda+\sigma^{2}\right)\left(H \nu_{1}\right. \\
& \left.+4 \pi^{2} v_{1}(\phi-1)+v_{2}\right)-4 \Lambda v_{0}^{7}\left(H v_{1}+4 \pi^{2} v_{1}(\phi-1)+v_{2}\right)+H \Lambda v_{0}^{10} \\
& +v_{0}^{4}\left(-4 \pi^{6}\left(H \Lambda(3 \phi-4)-8 \sigma^{2}(\phi-1)\right)+\pi^{4}\left(H \sigma^{2}(11-8 \phi)\right.\right. \\
& \left.\left.-16 R a_{n l}(\phi-1)\right)-3 \pi^{2} \Lambda v_{1}^{2}+48 \pi^{8} \Lambda(\phi-1)+v_{1}^{2}\left(-\sigma^{2}\right)\right) \\
& \left.\left.-3 \pi^{4} v_{1}^{2}\left(\pi^{2} \Lambda+\sigma^{2}\right)\right)\right) \text {. }
\end{aligned}
$$




\section{Rigid-Rigid, Isothermal Boundaries}

Following the procedure used for free-free, isothermal boundaries, we obtain the nanoliquid, solid and weighted average Nusselt number for rigid-rigid, isothermal boundaries as:

$$
\begin{aligned}
& N u_{n l}^{R R}=1+4 \pi \frac{F_{3}}{F_{4}}\left[1-\frac{1}{r}\right], \\
& N u_{s}^{R R}=1+4 \pi \frac{F_{3}}{F_{4}}\left(\frac{\gamma H}{4 \pi^{2}+\gamma H}\right)\left[1-\frac{1}{r}\right], \\
& N u_{w}^{R R}=1+4 \pi \frac{F_{3}}{F_{4}}\left(\frac{4 \pi^{2} \phi+\gamma H}{4 \pi^{2}+\gamma H}\right)\left[1-\frac{1}{r}\right],
\end{aligned}
$$

where $F_{3}, F_{4}$ and $\mathrm{r}$ are given by Eqs. (59), (114) and (120).

\section{Asymptotic Analysis for Small Values of H}

For small values of $\mathrm{H}$ with $R a_{n l}^{R R}$ given by Eq. (60) and $v$ given by Eq. (62), $N u_{w}^{R R}$ takes the form:

$$
N u_{w}^{R R}=G_{13}+G_{14} H+G_{15} H^{2}+\cdots,
$$

where

$$
\begin{aligned}
G_{13}= & +\frac{2 \pi \phi\left(2 F_{3}^{2} v_{0}^{2} R a_{n l}-\delta_{0}^{2} Q_{1}\right)}{F_{3} F_{4} v_{0}^{2} R a_{n l}}, \\
G_{14}= & \frac{1}{2 \pi F_{3} F_{4} v_{0}^{3} R a_{n l}}\left(\phi \left(F _ { 1 } \Lambda \left(v_{0}^{4}\left(\gamma \delta_{0}^{2} v_{0}-4 \pi^{2}\left(4 v_{1} v_{0}^{2}+v_{0}+2 \pi^{2} v_{1}\right)\right)\right.\right.\right. \\
& \left.+\mu_{1}^{4}\left(\gamma v_{0}^{3}+\pi^{2}(\gamma-4) v_{0}+8 \pi^{4} v_{1}\right)\right)+F_{2} \mu_{1}^{2}\left(2 \Lambda v _ { 0 } ^ { 3 } \left(\gamma v_{0}^{2}+\pi^{2}(\gamma\right.\right. \\
& \left.\left.\left.-8 v_{0} v_{1}-4\right)\right)+\sigma^{2}\left(\gamma v_{0}^{3}+\pi^{2}(\gamma-4) v_{0}+8 \pi^{4} v_{1}\right)\right)+F_{1} v_{0}^{3} \sigma^{2}\left(\gamma v_{0}^{2}\right. \\
& \left.\left.+\pi^{2}\left(\gamma-8 v_{0} v_{1}-4\right)\right)\right)-\gamma \delta_{0}^{2} v_{0}\left(F_{2} \mu_{1}^{2}\left(2 \Lambda v_{0}^{2}+\sigma^{2}\right)+F_{1} \Lambda\left(\mu_{1}^{4}+v_{0}^{4}\right)\right. \\
& \left.\left.+F_{1} v_{0}^{2} \sigma^{2}\right)-2 \gamma F_{3}^{2} v_{0}^{3} R a_{n l}(\phi-1)\right), \\
G_{15}= & \frac{1}{8 \pi F_{3} F_{4} v_{0}^{4} R a_{n l}}\left(8 v _ { 1 } v _ { 0 } \left(v _ { 0 } ^ { 4 } \left(2 \gamma F_{2} \Lambda \mu_{1}^{2}(\phi-1)+F_{1}\left(\pi^{2} \Lambda((\gamma-4)\right.\right.\right.\right. \\
& \left.\left.\phi-\gamma)+\gamma \sigma^{2}(\phi-1)\right)\right)-\pi^{2} \mu_{1}^{2}(\gamma(\phi-1)-4 \phi)\left(F_{1} \Lambda \mu_{1}^{2}+F_{2} \sigma^{2}\right) \\
& \left.+2 \gamma F_{1} \Lambda v_{0}^{6}(\phi-1)\right)-16 \pi^{2} \phi\left(F _ { 2 } \mu _ { 1 } ^ { 2 } \left(v_{1}^{2}\left(2 \Lambda v_{0}^{4}+3 \pi^{2} \sigma^{2}\right)\right.\right. \\
& \left.+2 v_{0} v_{2}\left(2 \Lambda v_{0}^{4}-\pi^{2} \sigma^{2}\right)\right)+F_{1}\left(\pi^{2} \Lambda \mu_{1}^{4}\left(3 v_{1}^{2}-2 v_{0} v_{2}\right)+v_{0}^{4}\left(v _ { 1 } ^ { 2 } \left(6 \Lambda v_{0}^{2}\right.\right.\right. \\
& \left.\left.\left.\left.+\pi^{2} \Lambda+\sigma^{2}\right)+2 v_{0} v_{2}\left(2 \Lambda v_{0}^{2}+\pi^{2} \Lambda+\sigma^{2}\right)\right)\right)\right)+\frac{1}{\pi^{2}}\left(\gamma v _ { 0 } ^ { 2 } \left(2 \gamma F_{3}^{2} v_{0}^{2}\right.\right. \\
& R a_{n l}(\phi-1)-\frac{1}{\delta_{0}^{2}}\left(\left(v_{0}^{2}(\phi-1)\left(\gamma v_{0}^{2}+2 \pi^{2}(\gamma-2)\right)+\pi^{4}(\gamma(\phi-1)\right.\right. \\
& \left.\left.\left.-20 \phi+4))\left(F_{2} \mu_{1}^{2}\left(2 \Lambda v_{0}^{2}+\sigma^{2}\right)+F_{1}\left(\Lambda\left(\mu_{1}^{4}+v_{0}^{4}\right)+v_{0}^{2} \sigma^{2}\right)\right)\right)\right)\right) .
\end{aligned}
$$




\section{Asymptotic Analysis for Large Values of H, i. e., $\frac{1}{H} \ll 1$}

For large values of $\mathrm{H}$ with $R a_{n l}^{R R}$ given by Eq. (72) and $v$ given by Eq. (74), $N u_{w}^{R R}$ takes the form:

$$
N u_{w}^{R R}=G_{16}+G_{17} \frac{1}{H}+G_{18} \frac{1}{H^{2}}+\cdots
$$

where

$$
\begin{aligned}
& G_{16}=1+\frac{2 \pi\left(2 \gamma F_{3}^{2} v_{0}^{2} R a_{n l}-(\gamma+1) \delta_{0}^{2} Q_{1}\right)}{\gamma F_{3} F_{4} v_{0}^{2} R a_{n l}}, \\
& G_{17}=\frac{1}{\gamma^{2} F_{3} F_{4} R a_{n l}}\left(2 \pi \left(\frac{1}{v_{0}^{2}}\left(\delta_{0}^{2}\left(\pi^{2}(-4(\gamma+1) \phi+4 \gamma+5)+v_{0}^{2}\right) Q_{1}\right)\right.\right. \\
& -\frac{1}{v_{0}^{3}}\left(2 \gamma ( \gamma + 1 ) \nu _ { 1 } \left(F_{2} \mu_{1}^{2}\left(2 \Lambda v_{0}^{4}-\pi^{2} \sigma^{2}\right)+F_{1}\left(v_{0}^{4}\left(2 \Lambda v_{0}^{2}+\pi^{2} \Lambda+\sigma^{2}\right)\right.\right.\right. \\
& \left.\left.\left.\left.\left.-\pi^{2} \Lambda \mu_{1}^{4}\right)\right)\right)+8 \pi^{2} \gamma F_{3}^{2} R a_{n l}(\phi-1)\right)\right), \\
& G_{18}=-\frac{1}{\gamma^{3} F_{3} F_{4} v_{0}^{4} R a_{n l}}\left(2 \pi \left(F _ { 2 } \mu _ { 1 } ^ { 2 } \left(v _ { 0 } ^ { 4 } \left(2 \gamma^{2}(\gamma+1) \Lambda v_{1}^{2}+2 \pi^{6} \Lambda(-4\right.\right.\right.\right. \\
& \left.(4 \gamma+5) \phi+16 \gamma+21)+\pi^{4} \sigma^{2}(-8(2 \gamma+3) \phi+16 \gamma+27)\right)+3 \pi^{2} \gamma^{2} \\
& (\gamma+1) \nu_{1}^{2} \sigma^{2}+\pi^{2} v_{0}^{6}\left(2 \pi^{2} \Lambda(-8(2 \gamma+3) \phi+16 \gamma+27)+\sigma^{2}(7-4 \phi)\right) \\
& +2 \gamma v_{0}^{5}\left(v_{1}\left(4 \pi^{2} \Lambda(2(\gamma+1) \phi-2 \gamma-3)-\sigma^{2}\right)+2 \gamma(\gamma+1) \Lambda v_{2}\right) \\
& -8 \gamma \Lambda v_{1} v_{0}^{7}+\pi^{6} v_{0}^{2} \sigma^{2}(-4(4 \gamma+5) \phi+16 \gamma+21)-2 \pi^{2} \gamma \nu_{0} \sigma^{2}\left(\pi^{2} \nu_{1}\right. \\
& \left.(4 \gamma(\phi-1)+4 \phi-5)+\gamma(\gamma+1) \nu_{2}\right)+v_{0}^{8}\left(2 \pi^{2} \Lambda(7-4 \phi)+\sigma^{2}\right) \\
& \left.+2 \Lambda v_{0}^{10}\right)+F_{1}\left(\Lambda \mu _ { 1 } ^ { 4 } \left(3 \pi^{2} \gamma^{2}(\gamma+1) v_{1}^{2}+\pi^{4} v_{0}^{4}(-8(2 \gamma+3) \phi+16 \gamma+27)\right.\right. \\
& +\pi^{6} v_{0}^{2}(-4(4 \gamma+5) \phi+16 \gamma+21)+2 \pi^{2} \gamma \nu_{0}\left(\pi^{2} \nu_{1}(-4(\gamma+1) \phi+4 \gamma+5)\right. \\
& \left.\left.-\gamma(\gamma+1) v_{2}\right)-2 \gamma v_{1} v_{0}^{5}+\pi^{2} v_{0}^{6}(7-4 \phi)+v_{0}^{8}\right)+v_{0}^{4}\left(v _ { 0 } ^ { 2 } \left(6 \gamma^{2}(\gamma+1) \Lambda v_{1}^{2}\right.\right. \\
& \left.+\pi^{4}\left(\pi^{2} \Lambda(-4(4 \gamma+5) \phi+16 \gamma+21)+\sigma^{2}(-8(2 \gamma+3) \phi+16 \gamma+27)\right)\right) \\
& +\gamma^{2}(\gamma+1) v_{1}^{2}\left(\pi^{2} \Lambda+\sigma^{2}\right)+\pi^{2} \nu_{0}^{4}\left(\pi^{2} \Lambda(-8(2 \gamma+3) \phi+16 \gamma+27)\right. \\
& \left.+\sigma^{2}(7-4 \phi)\right)+4 \gamma v_{0}^{3}\left(v_{1}\left(2 \pi^{2} \Lambda(2(\gamma+1) \phi-2 \gamma-3)-\sigma^{2}\right)\right. \\
& \left.+\gamma(\gamma+1) \Lambda \nu_{2}\right)+2 \gamma \nu_{0}\left(\pi ^ { 2 } \nu _ { 1 } \left(\pi^{2} \Lambda(4(\gamma+1) \phi-4 \gamma-5)+2 \sigma^{2}\right.\right. \\
& \left.(2(\gamma+1) \phi-2 \gamma-3))+\gamma(\gamma+1) \nu_{2}\left(\pi^{2} \Lambda+\sigma^{2}\right)\right)-6 \gamma \Lambda v_{1} v_{0}^{5}+\pi^{6} \sigma^{2} \\
& \left.\left.(-4(4 \gamma+5) \phi+16 \gamma+21)+v_{0}^{6}\left(\pi^{2} \Lambda(7-4 \phi)+\sigma^{2}\right)+\Lambda v_{0}^{8}\right)\right) \\
& \left.\left.+32 \pi^{4} \gamma F_{3}^{2} v_{0}^{4} R a_{n l}(\phi-1)\right)\right) \text {. }
\end{aligned}
$$

\section{Asymptotic Analysis for Large Values of $\gamma$, i. e., $\frac{1}{\gamma}<1$}

For large values of $\gamma$ with $R a_{n l}^{R R}$ given by Eq. (80) and $v$ given by Eq. (82), $N u_{w}^{R R}$ takes the form:

$$
N u_{w}^{R R}=G_{19}+G_{20} \frac{1}{\gamma}+G_{21} \frac{1}{\gamma^{2}}+\cdots,
$$


where

$$
\begin{aligned}
G_{19}=1 & +\frac{2 \pi \phi\left(2 F_{3}^{2} v_{0}^{2} R a_{n l}-\delta_{0}^{2} Q_{1}\right)}{F_{3} F_{4} v_{0}^{2} R a_{n l}} \\
G_{20}= & -\frac{1}{F_{3} F_{4} H v_{0}^{3} R a_{n l}}\left(2 \pi \left(F _ { 2 } \mu _ { 1 } ^ { 2 } \left(v _ { 0 } ( 2 \Lambda v _ { 0 } ^ { 2 } + \sigma ^ { 2 } ) \left(\pi^{2}\left(4 \delta_{0}^{2}(\phi-1)+H\right)\right.\right.\right.\right. \\
& \left.\left.+H v_{0}^{2}\right)-2 H v_{1}\left(\pi^{2} \sigma^{2}-2 \Lambda v_{0}^{4}\right)\right)+F_{1}\left(v _ { 0 } \left(\pi^{2}\left(4 \delta_{0}^{2}(\phi-1)+H\right)\right.\right. \\
& \left.+H v_{0}^{2}\right)\left(\Lambda\left(\mu_{1}^{4}+v_{0}^{4}\right)+v_{0}^{2} \sigma^{2}\right)+2 H v_{1}\left(-\pi^{2} \Lambda \mu_{1}^{4}+v_{0}^{4}\left(\pi^{2} \Lambda+\sigma^{2}\right)\right. \\
& \left.\left.\left.\left.+2 \Lambda v_{0}^{6}\right)\right)-8 \pi^{2} F_{3}^{2} v_{0}^{3} R a_{n l}(\phi-1)\right)\right) \\
G_{21}= & \frac{1}{F_{3} F_{4} H^{2} v_{0}^{4} R a_{n l}}\left(2 \pi \left(F _ { 2 } \mu _ { 1 } ^ { 2 } \left(H \left(v _ { 1 } \left(H v_{1}\left(2 \Lambda v_{0}^{4}+3 \pi^{2} \sigma^{2}\right)-2 v_{0}\right.\right.\right.\right.\right. \\
& \left.\left.\left(H+4 \pi^{2}(\phi-1)\right)\left(\pi^{2} \sigma^{2}-2 \Lambda v_{0}^{4}\right)\right)+2 H v_{0} v_{2}\left(2 \Lambda v_{0}^{4}-\pi^{2} \sigma^{2}\right)\right)-\delta_{0}^{2} v_{0}^{2} \\
& \left.\left(H v_{0}^{2}+\pi^{2}\left(H(5-4 \phi)+16 \pi^{2}(\phi-1)\right)\right)\left(2 \Lambda v_{0}^{2}+\sigma^{2}\right)\right)+F_{1}(H \\
& \left(\pi^{2} \Lambda \mu_{1}^{4}\left(v_{1}\left(3 H v_{1}-2 v_{0}\left(H+4 \pi^{2}(\phi-1)\right)\right)-2 H v_{0} v_{2}\right)+v_{0}^{4}\left(v _ { 1 } \left(2 v_{0}\right.\right.\right. \\
& \left.\left(H+4 \pi^{2}(\phi-1)\right)\left(2 \Lambda v_{0}^{2}+\pi^{2} \Lambda+\sigma^{2}\right)+H v_{1}\left(6 \Lambda v_{0}^{2}+\pi^{2} \Lambda+\sigma^{2}\right)\right) \\
& \left.\left.+2 H v_{0} v_{2}\left(2 \Lambda v_{0}^{2}+\pi^{2} \Lambda+\sigma^{2}\right)\right)\right)-\delta_{0}^{2} v_{0}^{2}\left(H v_{0}^{2}+\pi^{2}(H(5-4 \phi)\right. \\
& \left.\left.\left.\left.\left.+16 \pi^{2}(\phi-1)\right)\right)\left(\Lambda\left(\mu_{1}^{4}+v_{0}^{4}\right)+v_{0}^{2} \sigma^{2}\right)\right)+32 \pi^{4} F_{3}^{2} v_{0}^{4} \operatorname{Ra}(\phi-1)\right)\right) .
\end{aligned}
$$

\section{Asymptotic Analysis for Large Values of $\gamma H$}

For large values of $\gamma H$ with $R a_{n l}^{R R}$ given by Eq. (88) and $v$ given by Eq. (90), $N u_{w}^{R R}$ takes the form:

$$
N u_{w}^{R R}=G_{22}+G_{23} \frac{1}{\gamma H}+G_{24} \frac{1}{\gamma H^{2}}+\cdots,
$$

where

$$
\begin{aligned}
G_{22}=1 & +\frac{2 \pi \phi\left(2 F_{3}^{2} v_{0}^{2} R a_{n l}-\delta_{0}^{2} Q_{1}\right)}{F_{3} F_{4} v_{0}^{2} R a_{n l}}, \\
G_{23}= & -\frac{1}{F_{3} F_{4} v_{0}^{3} R a_{n l}}\left(2 \pi \left(F _ { 2 } \mu _ { 1 } ^ { 2 } \left(v_{0}\left(2 \Lambda v_{0}^{2}+\sigma^{2}\right)\left(\delta_{0}^{2} H+4 \pi^{2} \delta_{0}^{2}(\phi-1)\right)\right.\right.\right. \\
& \left.-2 v_{1}\left(\pi^{2} \sigma^{2}-2 \Lambda v_{0}^{4}\right)\right)+F_{1}\left(v _ { 0 } ( \delta _ { 0 } ^ { 2 } H + 4 \pi ^ { 2 } \delta _ { 0 } ^ { 2 } ( \phi - 1 ) ) \left(\Lambda\left(\mu_{1}^{4}+v_{0}^{4}\right)\right.\right. \\
& \left.\left.+v_{0}^{2} \sigma^{2}\right)+2 v_{1}\left(-\pi^{2} \Lambda \mu_{1}^{4}+v_{0}^{4}\left(\pi^{2} \Lambda+\sigma^{2}\right)+2 \Lambda v_{0}^{6}\right)\right) \\
& \left.\left.-8 \pi^{2} F_{3}^{2} v_{0}^{3} R a_{n l}(\phi-1)\right)\right), \\
G_{24}=- & \frac{1}{F_{3} F_{4} v_{0}^{4} R a_{n l}}\left(2 \pi \left(F _ { 2 } \mu _ { 1 } ^ { 2 } \left(2 v _ { 0 } ^ { 4 } \left(\Lambda\left(4 \pi^{2} \delta_{0}^{2} H \phi+v_{1}^{2}\right)-H\left(5 \pi^{4} \Lambda\right.\right.\right.\right.\right. \\
& \left.\left.+3 \pi^{2} \sigma^{2}\right)-8 \pi^{4}(\phi-1)\left(2 \pi^{2} \Lambda+\sigma^{2}\right)\right)+\pi^{2} v_{0}^{2} \sigma^{2}\left(4 \delta_{0}^{2} H \phi-5 \pi^{2} H\right. \\
& \left.-16 \pi^{4}(\phi-1)\right)-v_{0}^{6}\left(H\left(12 \pi^{2} \Lambda+\sigma^{2}\right)+32 \pi^{4} \Lambda(\phi-1)\right)+4 \Lambda v_{0}^{5}\left(v_{1}\right. \\
& \left.\left(H+4 \pi^{2}(\phi-1)\right)+v_{2}\right)-2 H \Lambda v_{0}^{8}-2 \pi^{2} v_{0} \sigma^{2}\left(v_{1}\left(H+4 \pi^{2}(\phi-1)\right)\right. \\
& \left.\left.+v_{2}\right)+3 \pi^{2} v_{1}^{2} \sigma^{2}\right)+F_{1}\left(H v _ { 0 } \left(2 v_{1}\left(v_{0}^{4}\left(2 \Lambda v_{0}^{2}+\pi^{2} \Lambda+\sigma^{2}\right)-\pi^{2} \Lambda \mu_{1}^{4}\right)\right.\right. \\
& \left.-v_{0}\left(-4 \pi^{2} \delta_{0}^{2} \phi+v_{0}^{4}+6 \pi^{2} v_{0}^{2}+5 \pi^{4}\right)\left(\Lambda\left(\mu_{1}^{4}+v_{0}^{4}\right)+v_{0}^{2} \sigma^{2}\right)\right)+v_{0}^{4}\left(v_{1}^{2}\right.
\end{aligned}
$$




$$
\begin{aligned}
& \left.\left(\pi^{2} \Lambda+\sigma^{2}\right)-16 \pi^{4}(\phi-1)\left(\Lambda \mu_{1}^{4}+\pi^{2} \sigma^{2}\right)\right)-16 \pi^{6} \Lambda \mu_{1}^{4} v_{0}^{2}(\phi-1) \\
& -2 \pi^{2} \Lambda \mu_{1}^{4} v_{0}\left(4 \pi^{2} v_{1}(\phi-1)+v_{2}\right)+3 \pi^{2} \Lambda \mu_{1}^{4} v_{1}^{2}+2 v_{0}^{6}\left(3 \Lambda v_{1}^{2}-8 \pi^{4}\right. \\
& \left.(\phi-1)\left(\pi^{2} \Lambda+\sigma^{2}\right)\right)+2 v_{0}^{5}\left(\pi^{2} \Lambda+\sigma^{2}\right)\left(4 \pi^{2} v_{1}(\phi-1)+v_{2}\right) \\
& \left.-16 \pi^{4} \Lambda v_{0}^{8}(\phi-1)+4 \Lambda v_{0}^{7}\left(4 \pi^{2} v_{1}(\phi-1)+v_{2}\right)\right) \\
& \left.\left.+32 \pi^{4} F_{3}^{2} v_{0}^{4} R a_{n l}(\phi-1)\right)\right)
\end{aligned}
$$

In the next section we discuss the results obtained from linear and non-linear stability analyses and make a few conclusions.

\section{Results and Discussion}

Linear and weakly nonlinear stability analyses of Rayleigh-Bénard convection for free- free and rigid-rigid, isothermal boundaries using LTNE model is studied. The thermophysical properties of ethylene-glycol [17] as the base liquid, copper [17] as the nanoparticle and 30\% glass fiber reinforced polycarbonate porous material [8] are considered. The thermophysical properties of porous material and ethylene-glycol copper saturated porous medium is calculated and the same is tabulated in Tables 1 and 2.

The effects of LTNE is found to be important only when $H>>0$ and $\gamma>>0$. The definitions of $\mathrm{H}$ and $\gamma$ both contain $\phi$ which makes it clear that when $\phi$ is close to 1 we have $\gamma H>>1$ and when $\phi$ is close to 0 then $\mathrm{H}$ is large. Table 11 gives us the exact meaning of $\mathrm{H}$ $\rightarrow 0$ and $\mathrm{H} \rightarrow \infty$ for large values of $\gamma$, for example, in the case of free-free boundaries with a value of 10 for $\gamma$, we see that $\mathrm{H} \rightarrow 0$ would mean any value of $\mathrm{H}$ equal to or smaller than 0.057 and the same is presented in Fig. 2. Similarly, $H \rightarrow \infty$ would mean any value of $H$ equal to or greater than 218.53, and this is presented in Fig. 3. From Table 11, it is clear that the exact meaning of $\mathrm{H} \rightarrow 0$ and $\mathrm{H} \rightarrow \infty$ predominantly depends on the value of $\gamma$ and less predominantly on the type of velocity boundary condition. In addition, Table 12 reveals the exact meaning of $\gamma \rightarrow \infty$ and $\gamma H \rightarrow \infty$ for large values of $H$. For example in the case of rigid-rigid boundaries with a value of 10 for $\mathrm{H}$, we see that $\gamma \rightarrow \infty$ would mean any value of $\gamma$ equal to or greater than 229.3 and the same is presented in Fig. 4. Similarly, $\gamma H \rightarrow \infty$ would mean any value of $\gamma H$ equal to or greater than 2292.98, and this is presented in Fig. 5. The comparison of exact and asymptotic solutions for small values of $\mathrm{H}$, large values of $\mathrm{H}, \gamma$ and $\gamma H$ in case of free-free and rigid-rigid boundaries is demonstrated in Tables 3, 4, $5,6,7,8,9$ and 10 and are in good agreement with each other as is to be expected.

From Figs. 6 and 7, it is clear that the effect of increasing $\mathrm{H}$ on the onset of convection is to stabilize the system. The value of the critical Rayleigh number of LTNE matches with that of LTE for large values of H. Thus the classical results of LTE case are obtained as a limiting case of LTNE for large values of H. The critical Rayleigh number of LTNE case is given by

$$
R a_{n l}^{L T E}=\left(\frac{\gamma}{1+\gamma}\right) R a_{n l}^{L T N E} .
$$

From Figs. 8 and 9, it is apparent that the effect of increasing $\gamma$ on the onset of convection is to destabilize the system and it is significant for small values of $\gamma$. Hence $\mathrm{H}$ and $\gamma$ have opposing influences on the onset of convection (Tables 11, 12).

We now discuss the non-linear stability results:

From Figs. 6 and 7 we observe the effect of $\mathrm{H}$ on heat transport. On increasing the value of $H$, weighted average Nusselt number decreases due to increase in rapid heat exchange 
between the phases and increase in residence time of heat in solid phase. Hence the effect of $H$ is to diminish the amount of heat transport.

The effect of increasing the value of $\gamma$ enhances the amount of heat transport due to high thermal conductivity of the nanoliquid and low thermal conductivity of the porous medium which is shown in Figs. 8 and 9. Thus $\mathrm{H}$ and $\gamma$ have opposing effects on the heat transport.

The critical Rayleigh number increases with increase in $\Lambda$ and this result is depicted in Figs. 10 and 11. Increase in the value of $\Lambda$ signifies decrease in the value of permeability and hence less space is available for the nanoliquid to flow. Hence this results in delayed onset of convection and decrease in heat transport.

The critical Rayleigh number increases with increase in $\sigma^{2}$ and this is shown in Figs. 12 and 13. The Nusselt number decreases with increase in $\sigma^{2}$ which is also depicted in Figs. 12 and 13. The low thermal conductivity of the porous medium slows down the flow and diminishes the heat transport.

From Figs. 14 and 15, it is clear that the onset of convection is advanced in the case of LTNE compared to that in the case of LTE for both free-free and rigid-rigid boundaries. Also, the amount of heat transport is more in the case of LTNE than in the case of LTE.

The onset of convection is delayed in the case of rigid-rigid boundaries than in the case free-free boundaries and this result is depicted in Fig. 16.

In the present study, the value of critical Rayleigh number is large due to the presence of porous medium which has a very low thermal conductivity and retains the heat. Experimental investigation of the above study has been carried out by Katto and Masuoka [9].

\section{Conclusion}

(a) The critical value of Rayleigh number and Nusselt number of the nanoliquid saturated porous medium vary as shown below.

$$
R a_{n l_{c}}^{F F}<R a_{n l_{c}}^{F F}, N u_{w}^{F F}>N u_{w}^{R R}
$$

(b) The effect of LTNE ceases both in the presence and absence of rotation for small values of $\mathrm{H}$, large values of $\mathrm{H}, \gamma$ and $\gamma H$.

(c) $R a_{n l_{c}}^{L T N E}<R a_{n l_{c}}^{L T E}, N u_{w}^{L T N E}>N u_{w}^{L T E}$

(d) The effect of increasing $\Lambda, \sigma^{2}$ and $\mathrm{H}$ is to stabilize the system whereas the effect of increasing $\gamma$ is to destabilize the system.

(i) $\frac{d}{d \Lambda}\left(R a_{n l_{c}}\right)>0, \frac{d}{d \Lambda}\left(N u_{w}\right)<0$.

(ii) $\frac{d}{d \sigma^{2}}\left(R a_{n l_{c}}\right)>0, \frac{d}{d \sigma^{2}}\left(N u_{w}\right)<0$.

(iii) $\frac{d}{d H}\left(R a_{n l_{c}}\right)>0, \frac{d}{d H}\left(N u_{w}\right)<0$.

(iv) $\frac{d}{d \gamma}\left(R a_{n l_{c}}\right)<0, \frac{d}{d \gamma}\left(N u_{w}\right)>0$.

(e) The onset of convection is delayed in the case of rigidrigid, isothermal boundaries than that in the case of freefree, isothermal boundaries which in turn leads to a diminished heat transport situation in the former case compared to the latter.

(f) Asymptotic and Galerkin solutions are found to be in good agreement with each other. 


\section{References}

1. Agarwal, S., Bhadauria, B.S.: Natural convection in a nanofluid saturated rotating porous layer with thermal non-equilibrium model. Transp. Porous Media 90, 627-654 (2011a)

2. Agarwal, S., Bhadauria, B.S., Siddheshwar, P.G.: Thermal instability of a nanofluid saturating a rotating anisotropic porous medium. Spec. Top. Rev. Porous Media 2(1), 53-64 (2011)

3. Banu, N., Rees, D.A.S.: Onset of Bénard convection using a thermal non-equilibrium model. Int. J. Heat Mass Transf. 45, 2221-2228 (2002)

4. Barletta, A., Celli, M., Lagziri, H.: Instability of a horizontal porous layer with local thermal nonequilibrium: effects of free surface and convective boundary conditions. Int. J. Heat Mass Transf. 89, 75-89 (2015)

5. Celli, M., Lagziri, H., Bezzazi, M.: Local thermal non-equilibrium effects in the Horton-Rogers-Lapwood problem with a free surface. Int. J. Therm. Sci. 116, 254-264 (2017)

6. Chandrasekhar, S.: Hydrodynamic and Hydromagnetic Stability. Clarendon Press, London (1961)

7. Chandrasekhar, S., Reid, W.H.: On the expansion of functions which satisfy four boundary conditions. Proc. Natl. Acad. Sci. U.S.A. 43, 521-527 (1957)

8. https://www.matbase.com/material-categories/natural-and-synthetic-composites/polymer-matrix-comp osites-pmc/reinforced-polymers/material-properties-of-polycarbonate-30-percent-glass-fiber-reinforce d-pc-gf30.html

9. Katto, Y., Masuoka, T.: Criterion for the onset of convective flow in a fluid in a porous medium. Int. J. Heat Mass Transf. 10(3), 297-309 (1967)

10. Nagata, M.: Bifurcations at the Eckhaus points in two-dimensional Rayleigh-Bénard convection. Phys. Rev. E 52, 6141-6145 (1995)

11. Nield, D.A., Bejan, A.: Convection in Porous Media. Springer, New York (2006)

12. Nield, D.A., Kuznetsov, A.V.: Local thermal non-equilibrium and heterogeneity effects on the onset of convection in a layered porous medium. Transp. Porous Media 102, 1-13 (2014)

13. Nield, D.A., Kuznetsov, A.V., Barletta, A., Celli, M.: The onset of convection in a sloping layered porous medium: effects of local thermal non-equilibrium and heterogeneity. Transp. Porous Med. 14, 87-97 (2016)

14. Postelnicu, A.: The onset of a Darcy-Brinkman convection using a thermal nonequilibrium model. Part II. Int. J. Therm. Sci. 47, 1587-1594 (2008)

15. Postelnicu, A., Rees, D.A.S.: The onset of Darcy-Brinkman convection in a porous layer using a thermal nonequlibrium model—part I: stress free boundaries. Int. J. Energy Res. 27, 961-973 (2003)

16. Rees, D.A.S., Pop, I.: Local thermal non-equilibrium in porous medium convection. Transp. Porous Media III, 147-173 (2005)

17. Siddheshwar, P.G., Meenakshi, N.: Amplitude equation and heat transport for Rayleigh-Bénard convection in Newtonian liquids with nanoparticles. Int. J. Appl. Comput. Math. 2, 1-22 (2015)

18. Siddheshwar, P.G., Siddabasappa, C.: Effect of Coriolis force on Brinkman-Bénard convection with late effects. Math. Sci. Int. Res. J. 6(1), 53-56 (2017)

19. Siddheshwar, P.G., Siddabasappa, C.: Linear and weakly nonlinear stability analyses of two-dimensional, steady Brinkman-Bénard convection using local thermal non-equilibrium model. Transp. Porous Media 120(3), 605-631 (2017)

20. Straughan, B.: Global nonlinear stability in porous convection with a thermal non-equilibrium model. Proc. R. Soc. Lond. A. 462, 409-418 (2006)

Publisher's Note Springer Nature remains neutral with regard to jurisdictional claims in published maps and institutional affiliations. 